\title{
Conserving algorithms for frictionless and full stick friction dynamic contact problems using the direct elimination method
}

\author{
C. Agelet de Saracibar ${ }^{1,3}$, D. Di Capua ${ }^{2,3}$ \\ ${ }^{1}$ ETS Ingenieros de Caminos, Canales y Puertos, Universidad Politécnica de Cataluña (UPC), \\ Edificio C1, Campus Norte, UPC, Jordi Girona 1-3, 08034 Barcelona, Spain \\ ${ }^{2}$ Escola d'Enginyeria Barcelona Est (EEBE), Universidad Politécnica de Cataluña (UPC), \\ Av. d'Eduard Maristany, 10-14, 08930 Barcelona, Spain \\ ${ }^{3}$ International Center for Numerical Methods in Engineering (CIMNE), Gran Capitán s/n, 08034 Barcelona, Spain \\ agelet@cimne.upc.edu, dicapua@cimne.upc.edu,
}

First version on 1 April 2017

Revised on 15 June 2017

Submitted to

International Journal for Numerical Methods in Engineering 


\title{
Conserving algorithms for frictionless and full stick friction dynamic contact problems using the direct elimination method
}

\author{
C. Agelet de Saracibar ${ }^{1,3}$, D. Di Capua ${ }^{2,3}$ \\ ${ }^{1}$ ETS Ingenieros de Caminos, Canales y Puertos, Universidad Politécnica de Cataluña (UPC), \\ Edificio C1, Campus Norte, UPC, Jordi Girona 1-3, 08034 Barcelona, Spain \\ ${ }^{2}$ Escola d'Enginyeria Barcelona Est (EEBE), Universidad Politécnica de Cataluña (UPC), \\ Av. d'Eduard Maristany, 10-14, 08930 Barcelona, Spain \\ ${ }^{3}$ International Center for Numerical Methods in Engineering (CIMNE), Gran Capitán s/n, 08034 Barcelona, Spain \\ agelet@cimne.upc.edu, dicapua@cimne.upc.edu,
}

\section{Summary}

In this paper, conserving time-stepping algorithms for frictionless and full stick friction dynamic contact problems are presented. Time integration algorithms for frictionless and full stick friction dynamic contact problems have been designed in order to preserve the conservation of key discrete properties satisfied at the continuum level. Energy and energy-momentum preserving algorithms for frictionless and full stick friction dynamic contact problems, respectively, have been designed and implemented within the framework of the direct elimination method, avoiding the drawbacks linked to the use of penalty-based or Lagrange multipliers methods. An assessment of the performance of the resulting formulation is shown in a number of selected and representative numerical examples, under full stick friction and slip frictionless contact conditions. Conservation of key discrete properties exhibited by the time stepping algorithm is shown.

Keywords: contact mechanics, full stick friction, frictionless, finite elements, dynamics, conserving algorithms, direct elimination method

\section{Introduction, motivation and goals}

Numerical analysis of contact problems has been one of the hot research topics of interest over the last decades. Contact problems arise in many applications, such as in crashworthiness, projectile impact, and material forming processes, i.e. sheet metal forming, bulk forming, casting, friction stir welding, cutting, powder compaction, and additive manufacturing. Despite the important progresses achieved in computational contact mechanics, the numerical simulation of contact problems is still nowadays a complex task, mainly due to the highly nonlinear nature of the problem, potentially involving fully nonlinear kinematics, finite strains, 
large slips, nonlinear boundary conditions, complex frictional behavior phenomena, thermomechanical contact, etc.

Mathematically, the numerical analysis of a contact problem amounts to finding the solution of an Initial Boundary Value Problem (IBVP) within a constrained solution space. The variational formulation of a contact problem includes restrictions on the admissible variations in the tangent solution space induced by the contact constraints, yielding to Variational Inequalities (VI). See, for instance, Kikuchi \& Oden (1988) [22] and Duvaut \& Lions (1972) [10]. The solution of contact problems has been typically tackled using two approaches: Lagrange multipliers and penalty-based methods. A good overview of those approaches can be found in the classical books written by Laursen (2002) [26] and Wriggers (2006) [40]. An alternative approach to solve contact problems, the direct elimination algorithm (DEA), is briefly addressed by Wriggers [40].

Within the Lagrange multipliers method the kinematical contact constraints are exactly satisfied. On the other hand, Lagrange multiplier methods are cumbersome to implement and the total number of equations increases and changes during the solution process. Within the penalty-based methods, a regularization of the contact constraints, using penalty or augmented Lagrangian approaches, allows us to bypass the need to find a solution within a constrained solution space and provides a very convenient displacement driven contact formulation. Well known drwabacks linked to the penalty method are the penalty sensitivity and possible illconditioning of the system of equations. To avoid those main drawbacks exhibited by the penalty method, while retaining his advantages, the augmented Lagrangian method, using Uzawa's algorithm, has been used as an alternative regularization procedure [26],[40]. The DEA for quasi-static and dynamic frictionless and full stick friction contact problems has been addressed by Di Capua \& Agelet de Saracibar (2015) [9]. Using the DEA, Lagrange multipliers and penalty-based regularized formulations of the contact problem, such as penalty or Augmented Lagrangian methods, are fully avoided and the contact constraints are directly imposed yielding a modified set of residual equations.

At the contact discrete level, two classical approaches, node-to-segment (NTS) and segment-to-segment (STS) are widely used. NTS is one of the most popular approaches to tackle contact problems, particularly within the framework of large deformations. This popularity mainly comes due to the simplicity of its implementation, compared to other methods. Key references on NTS contact methods, addressing penalty, Lagrange multipliers, and augmented Lagrangian methods, are the now classical books written by Laursen (2002) [26] and Wriggers (2006) [40]. Its popularity has been maintained despite the fact that, as it is well know now, NTS methods do not pass the patch test and exhibits poor convergence properties [28]. In particular, local errors at the contact surface do not necessarily diminish when the mesh is refined [11]. To avoid those drawbacks inherent to NTS methods, a new family of STS contact methods, called mortar methods, was developed and it is now well established for contact problems. For applications of the mortar method to two-dimensional contact problems, see McDevitt \& Laursen (2000) [27], Yang [41], Flemish et al. (2005) [13], and Fischer \& Wriggers (2005) [12]. A STS mortar contact method for quadratic elements is proposed by Puso et al. (2008) [32]. Three-dimensional applications of the mortar method can be find in Puso \& Laursen [30],[31]. Dual active set strategies based on the mortar method have been presented by 
Wohlmuth (2000) [39], Hüeber \& Wohlmuth (2005) [21], Brunssen et al. (2007) [8], and Popp et al. (2010) [29].

Energy-momentum algorithms for non-linear elastodynamics problems have been first developed by Simo \& Tarnow [34] and Simo et al. [35]. Further improvements for general models in nonlinear elasticity were developep by Gonzalez [14]. First applications of the energy-momentum scheme to unilateral contact constraints within the framework of the NTS methods can be found in Laursen \& Chawla (1997) [24] and in Armero \& Petocz $(1998,1999)$ [3],[4]. Further developments, also within the NTS framework, can be found in Laursen \& Love (2002) [25], Hauret \& Le Tallec (2006) [16], Haikal \& Hjelmstad (2007) [15], Betsch \& Hesch (2007) [7], and Hesch \& Betsch (2011) [18]. Applications of the energy-momentum scheme using the mortar method can be found in Hesch \& Betsch (2010) [19] for transient 3d domain decomposition problems, in Hesch \& Betsch (2009) [20] for two-dimensional contact problems, and in Hesch \& Betsch (2011) [17] for three-dimensional contact problems.

This work is a follow-up of the one published by Di Capua \& Agelet de Saracibar (2015) [9] where the main aspects of the DEA for quasi-static and dynamic contact problems were introduced. Here now the focus is placed on the development, implementation and validation of energy-momentum conserving schemes formulated within the framework of the direct elimination method. In particular conserving algorithms are presented for the two cases of interest in dynamic contact problems: a energy conserving scheme for the full stick frictional case, and an energy-momentum conserving scheme for the slip frictionless case. Finite deformation kinematics is considered. On the other hand, a more convenient and natural formulation of the direct elimination method for the slip frictionless case is derived. This formulation is given now in terms of the contravariant components of the iso-parametric coordinates of the incremental slip of the closest-point-projection on the covariant basis vectors evaluated at the mid-point configuration.

The outline of the paper is as follows. In Section 2, the main aspects of the contact problem at a continuum setting are briefly presented. Section 3 deals with the finite element formulation of the contactless problem. Section 4 deals with the main aspects of the finite element formulation and numerical solution of the full stick and slip frictionless contact cases, using the proposed DEA. Section 5 shows the key conservation properties exhibited by the time stepping algorithms used in the numerical analysis of dynamic contact problems, for both the full stick and slip frictionless cases. Finally, Section 6 deals with an assessment of the energymomentum conserving schemes proposed within the framework of the DEA, through a number of representative numerical examples, under full stick and slip frictionless dynamic contact conditions. The paper concludes with some final remarks. An Appendix, including the computation of the variations of the covariant tangent vectors $\tau_{\xi, n+\alpha}$ and $\tau_{\eta, n+\alpha}$ at the configuration $n+\alpha$ of the closest-point-projection on the master surface, has been added.

\section{Continuum formulation of the contact mechanics problem}

\subsection{Local formulation}


Let $2 \leq n_{\text {dim }} \leq 3$ be the space dimension and $\mathbb{I}:=[0, T] \subset \mathbb{R}_{+}$the time interval of interest. Let the open sets $\Omega^{(1)} \subset \mathbb{R}^{n_{\mathrm{dim}}}$ and $\Omega^{(2)} \subset \mathbb{R}^{n_{\mathrm{dim}}}$, with smooth boundaries $\partial \Omega^{(1)}$ and $\partial \Omega^{(2)}$ and closures $\bar{\Omega}^{(1)}=\Omega^{(1)} \cup \partial \Omega^{(1)}$ and $\bar{\Omega}^{(2)}=\Omega^{(2)} \cup \partial \Omega^{(2)}$, be the reference placement of two continuum bodies $\mathcal{B}^{(1)}$ and $\mathcal{B}^{(2)}$.

For each body $\mathcal{B}^{(i)}$ we denote by $\mathbf{X}^{(i)} \in \bar{\Omega}^{(i)}$ the vector position of the material particles at the reference configuration, $\varphi^{(i)}: \bar{\Omega}^{(i)} \times \mathbb{I} \rightarrow \mathbb{R}^{n_{\text {dim }}}$ the orientation preserving deformation maps, $\mathbf{V}^{(i)}:=\partial_{t} \boldsymbol{\varphi}^{(i)}$ the material velocities, $\mathbf{u}^{(i)}:=\boldsymbol{\varphi}^{(i)}-\mathbf{X}^{(i)}$ the material displacements, $\rho_{0}^{(i)}$ the reference mas densities, and $\mathbf{F}^{(i)}:=\mathrm{GRAD} \varphi^{(i)}$ the deformation gradients, where GRAD denotes the material gradient operator. For each time $t \in \mathbb{I}$, the mapping $t \in \mathbb{I} \mapsto \boldsymbol{\varphi}_{t}^{(i)}:=\boldsymbol{\varphi}^{(i)}(\cdot, t)$ represents a one-parameter family of configurations indexed by time $t$, which maps the reference placement of body $\mathcal{B}^{(i)}$ onto its current placement $\mathcal{S}_{t}^{(i)}: \varphi_{t}^{(i)}\left(\mathcal{B}^{(i)}\right) \subset \mathbb{R}^{n_{\text {dim }}}$. The current placement of particles $\mathbf{X}^{(i)} \in \bar{\Omega}^{(i)}$ at time $t \in \mathbb{I}$ is denoted as $\mathbf{X}^{(i)}:=\varphi^{(i)}\left(\mathbf{X}^{(i)}, t\right)$.

We will asume that no contact forces are present between the two bodies at the reference configuration. Subsequent configurations cause the two bodies to physically come into contact and produce contact interactive forces during some portion of the time interval of interest $\mathbb{I}:=[0, T] \subset \mathbb{R}_{+}$.

For each body $\mathcal{B}^{(i)}$ we will consider the following partitions of the boundary $\partial \Omega^{(i)}=\partial_{u} \Omega^{(i)} \cup \partial_{\sigma} \Omega^{(i)} \cup \partial_{c} \Omega^{(i)}$, where $\partial_{u} \Omega^{(i)}, \partial_{\sigma} \Omega^{(i)}$ and $\Gamma_{c}^{(i)}:=\partial_{c} \Omega^{(i)}$ represent the prescribed displacements, prescribed nominal tractions and contact boundaries, respectively, such that the conditions $\varnothing=\partial_{u} \Omega^{(i)} \cap \partial_{\sigma} \Omega^{(i)}, \varnothing=\partial_{u} \Omega^{(i)} \cap \partial_{c} \Omega^{(i)}$ and $\varnothing=\partial_{\sigma} \Omega^{(i)} \cap \partial_{c} \Omega^{(i)}$, hold.

The local material form of the momentum balance equation, prescribed traction and prescribed displacement boundary conditions, and initial conditions for body $\mathcal{B}^{(i)}$ take the form,

$$
\begin{aligned}
\operatorname{DIV} \mathbf{P}^{(i)}+\rho_{0}^{(i)} \mathbf{B}^{(i)} & =\rho_{0}^{(i)} \dot{\mathbf{V}}^{(i)} & & \text { in } \Omega^{(i)} \times[0, T] \\
\mathbf{P}^{(i)} \mathbf{N}^{(i)} & =\overline{\mathbf{T}}^{(i)} & & \text { on } \partial_{\sigma} \Omega^{(i)} \times[0, T] \\
\mathbf{u}^{(i)} & =\overline{\mathbf{u}}^{(i)} & & \text { on } \partial_{u} \Omega^{(i)} \times[0, T] \\
\mathbf{V}^{(i)} & =\mathbf{V}_{0}^{(i)} & & \text { in } \Omega^{(i)}
\end{aligned}
$$

where $\mathbf{P}^{(i)}:=\mathbf{F}^{(i)} \mathbf{S}^{(i)}$ is the First Piola-Kirchhoff stress tensor for body $\mathcal{B}^{(i)}, \mathbf{S}^{(i)}$ is the second Piola-Kirchhoff stress tensor for body $\mathcal{B}^{(i)}$, DIV denotes the material divergence operator, $\mathbf{N}^{(i)}$ is the outward unit normal to the boundary $\partial_{\sigma} \Omega^{(i)}, \overline{\mathbf{T}}^{(i)}$ is the prescribed nominal traction vector on the boundary $\partial_{\sigma} \Omega^{(i)}, \overline{\mathbf{u}}^{(i)}$ is the prescribed displacement vector on the boundary $\partial_{u} \Omega^{(i)}$, and $\mathbf{V}_{0}^{(i)}$ is the initial velocity in $\Omega^{(i)}$. The superimposed dot refers to the material time derivative.

Assuming a linear Saint-Venant Kirchhoff elastic constitutive model for the body $\mathcal{B}^{(i)}$, the free energy per unit of mass can be expressed as,

$$
\Psi^{(i)}\left(\mathbf{E}^{(i)}\right)=\frac{1}{2 \rho_{0}} \mathbf{E}^{(i)}: \mathbb{C}^{(i)}: \mathbf{E}^{(i)}
$$


where $\mathbb{C}^{(i)}$ is the constant fourth order elastic constitutive tensor and $\mathbf{E}^{(i)}$ is the GreenLagrange strain tensor defined as,

$$
\mathbf{E}^{(i)}:=\frac{1}{2}\left(\mathbf{F}^{(i) T} \mathbf{F}^{(i)}-\mathbf{1}\right)
$$

where $\mathbf{1}$ is the second order unit tensor.

Following a standard procedure, using the Clausius-Planck inequality and following Coleman's method, the Second Piola-Kirchhoff stress tensor takes the form [38],

$$
\mathbf{S}^{(i)}:=\rho_{0} \frac{\partial \Psi^{(i)}\left(\mathbf{E}^{(i)}\right)}{\partial \mathbf{E}^{(i)}}=\mathbb{C}^{(i)}: \mathbf{E}^{(i)}
$$

and the First Piola-Kirchhoff stress tensor is computed as $\mathbf{P}^{(i)}:=\mathbf{F}^{(i)} \mathbf{S}^{(i)}$.

\subsection{Local formulation of the contact problem}

Using the classical slave-master formulation of contact mechanics [1],[2],[23],[26],[40] let us denote the contact surfaces $\Gamma_{c}^{(1)}$ and $\Gamma_{c}^{(2)}$ as slave and master contact surfaces, respectively. Particles of the slave and master contact surfaces will be denoted as slave particles and master particles, respectively.

Let us consider a slave particle $\mathbf{X}^{(1)} \in \Gamma_{c}^{(1)}$, being $\mathbf{x}^{(1)}=\boldsymbol{\varphi}^{(1)}\left(\mathbf{X}^{(1)}, t\right) \in \gamma_{c}^{(1)}$ its current spatial vector position of the slave particle at time $t \in \mathbb{I}$, and $\overline{\mathbf{y}}\left(\mathbf{X}^{(1)}, t\right) \in \gamma_{c}^{(2)}$ its closest-pointprojection onto the spatial configuration $\gamma_{c}^{(2)}$ of the master surface $\Gamma_{c}^{(2)}$ at time $t \in \mathbb{I}$, defined as,

$$
\begin{aligned}
& \overline{\mathbf{y}}\left(\mathbf{X}^{(1)}, t\right):=\varphi^{(2)}\left(\overline{\mathbf{Y}}\left(\mathbf{X}^{(1)}, t\right), t\right) \\
& \overline{\mathbf{Y}}\left(\mathbf{X}^{(1)}, t\right):=\arg \min _{\mathbf{X}^{(2)} \in \Gamma_{c}^{(2)}}\left\|\varphi^{(1)}\left(\mathbf{X}^{(1)}, t\right)-\boldsymbol{\varphi}^{(2)}\left(\mathbf{X}^{(2)}, t\right)\right\|
\end{aligned}
$$

The contact normal gap function $g_{N}\left(\mathbf{X}^{(1)}, t\right)$ for a slave particle $\mathbf{X}^{(1)} \in \Gamma_{c}^{(1)}$ at time $t \in \mathbb{I}$ is defined as,

$$
g_{N}\left(\mathbf{X}^{(1)}, t\right):=\left(\varphi^{(1)}\left(\mathbf{X}^{(1)}, t\right)-\varphi^{(2)}\left(\overline{\mathbf{Y}}\left(\mathbf{X}^{(1)}, t\right), t\right)\right) \cdot \mathbf{n}
$$

where $\mathbf{n}$ is the outward unit normal to the spatial configuration of the master surface at the closest-point-projection. Assuming enough smooth contact surfaces, when a slave particle $\mathbf{X}^{(1)} \in \Gamma_{c}^{(1)}$ comes into contact with a master surface $\gamma_{c}^{(2)}$, it is assumed that the following condition holds,

$$
\mathbf{n}=\mathbf{n}^{(2)}\left(\overline{\mathbf{Y}}\left(\mathbf{X}^{(1)}, t\right), t\right)=-\mathbf{n}^{(1)}\left(\mathbf{X}^{(1)}, t\right)
$$

The nominal frictional contact traction vector at a material particle on the contact surface $\Gamma_{c}^{(i)}:=\partial_{c} \Omega^{(i)}$ is given by, 


$$
\mathbf{T}^{(i)}:=\mathbf{P}^{(i)} \mathbf{N}^{(i)}=\mathbf{F}^{(i)} \mathbf{S}^{(i)} \mathbf{N}^{(i)}
$$

where $\mathbf{N}^{(i)}$ is the outward unit normal to the contact surface $\Gamma_{c}^{(i)}:=\partial_{c} \Omega^{(i)}$ on the material configuration of the body $\mathcal{B}^{(i)}$. The nominal frictional contact vector $\mathbf{T}^{(1)}\left(\mathbf{X}^{(1)}, t\right)$ at a slave particle $\mathbf{X}^{(1)} \in \Gamma_{c}^{(1)}$ at time $t \in \mathbb{I}$ can be additively split as,

$$
\mathbf{T}^{(1)}\left(\mathbf{X}^{(1)}, t\right):=\mathbf{t}_{N}\left(\mathbf{X}^{(1)}, t\right)-\mathbf{t}_{T}\left(\mathbf{X}^{(1)}, t\right)=t_{N}\left(\mathbf{X}^{(1)}, t\right) \mathbf{n}+(\mathbf{1}-\mathbf{n} \otimes \mathbf{n}) \mathbf{T}^{(1)}\left(\mathbf{X}^{(1)}, t\right)
$$

where $t_{N}\left(\mathbf{X}^{(1)}, t\right)$ and $\mathbf{t}_{T}\left(\mathbf{X}^{(1)}, t\right)$ are the nominal contact pressure and nominal frictional tangent traction vector, respectively.

The unilateral contact constrained problem can be characterized by the following KuhnTucker and contact persistency conditions [1],[2],[23],[26],[40]:

$$
\begin{gathered}
g_{N}\left(\mathbf{X}^{(1)}, t\right) \geq 0, \quad t_{N}\left(\mathbf{X}^{(1)}, t\right) \geq 0, \quad t_{N}\left(\mathbf{X}^{(1)}, t\right) g_{N}\left(\mathbf{X}^{(1)}, t\right)=0 \\
\text { if } \quad g_{N}\left(\mathbf{X}^{(1)}, t\right)=0 \quad \text { then } \quad t_{N}\left(\mathbf{X}^{(1)}, t\right) \dot{g}_{N}\left(\mathbf{X}^{(1)}, t\right)=0
\end{gathered}
$$

where

$$
\dot{g}_{N}\left(\mathbf{X}^{(1)}, t\right):=\left(\mathbf{V}^{(1)}\left(\mathbf{X}^{(1)}, t\right)-\mathbf{V}^{(2)}\left(\overline{\mathbf{Y}}\left(\mathbf{X}^{(1)}, t\right), t\right)\right) \cdot \mathbf{n}
$$

\subsection{Variational formulation}

The variational form of the momentum balance equation for a problem involving contact between two bodies $\mathcal{B}^{(1)}$ and $\mathcal{B}^{(2)}$ can be written as [1],[2],[23],[26],[40]:

$$
\begin{aligned}
& \sum_{i=1}^{2}\left(\rho_{0}^{(i)} \dot{\mathbf{V}}^{(i)}, \boldsymbol{\eta}^{(i)}\right)+\sum_{i=1}^{2}\left(\mathbf{P}^{(i)}, \operatorname{GRAD} \boldsymbol{\eta}^{(i)}\right)=\sum_{i=1}^{2}\left(\rho_{0}^{(i)} \mathbf{B}^{(i)}, \boldsymbol{\eta}^{(i)}\right) \\
& \quad+\sum_{i=1}^{2}\left(\overline{\mathbf{T}}^{(i)}, \boldsymbol{\eta}^{(i)}\right)_{\partial_{\sigma} \Omega^{(i)}}+\left(\mathbf{T}^{(1)}, \boldsymbol{\eta}^{(1)}-\boldsymbol{\eta}^{(2)}\right)_{\Gamma_{c}^{(1)}}
\end{aligned}
$$

for any admissible variations $\boldsymbol{\eta}^{(i)}: \Omega^{(i)} \rightarrow \mathbb{R}^{n_{\text {dim }}}$ such that $\boldsymbol{\eta}^{(i)}=\mathbf{0}$ on $\partial_{u} \Omega^{(i)}$.

\subsection{Linear momentum, angular momentum and total energy of the system}

The material form of the linear momentum $\mathbf{L}$ and angular momentum $\mathbf{J}$ of the system are given by:

$$
\begin{aligned}
\mathbf{L} & :=\sum_{i=1}^{2} \int_{\Omega^{(i)}} \rho_{0}^{(i)} \mathbf{V}^{(i)} d V \\
\mathbf{J} & :=\sum_{i=1}^{2} \int_{\Omega^{(i)}} \mathbf{x}^{(i)} \times \rho_{0}^{(i)} \mathbf{V}^{(i)} d V
\end{aligned}
$$

The total energy of a system $E$ can be additively split as:

$$
E:=K+W+\Pi^{e x t}
$$

where $K, W$ and $\Pi^{e x t}$ are the kinetic energy, elastic strain energy, and potential energy for the external loads, respectively, given by: 


$$
\begin{aligned}
K & :=\sum_{i=1}^{2} \int_{\Omega^{(i)}} \frac{1}{2} \rho_{0}^{(i)} \mathbf{V}^{(i)} \cdot \mathbf{V}^{(i)} d V \\
W & :=\sum_{i=1}^{2} \int_{\Omega^{(i)}} \rho_{0}^{(i)} \Psi^{(i)} d V \\
\Pi^{e x t} & :=\sum_{i=1}^{2} \Pi^{e x t(i)}
\end{aligned}
$$

where $\Pi^{\text {ext }(i)}$ is the potential energy for the external forces of body $\mathcal{B}^{(i)}$.

It can be shown [5],[6],[14],[24],[34],[35],[36],[37] that the linear momentum $\mathbf{L}$, angular momentum $\mathbf{J}$, and total energy $E$ are conserved for a homogeneous Neumann boundary problem, characterized by zero body forces and zero natural boundary conditions, yielding zero potential energy for the external loads, i.e. $\Pi^{e x t}=0$. The total energy $E$ is also conserved if the external loading is conservative. A typical case of conservative external loading is the case of gravitational body forces and constant prescribed nominal tractions.

\section{Finite element formulation of the continuum problem without frictional contact constraints}

Let us consider first the finite element discretization of the dynamic continuum problem without frictional contact constraints. Using a standard finite element discretization, the material vector position $\mathbf{X}^{(i)} \in \bar{\Omega}_{h}^{(i)}$, displacement $\mathbf{u}^{(i)}$ and material velocitie $\mathbf{V}^{(i)}$ of the particles of body $\mathcal{B}^{(i)}$ , take the form,

$$
\mathbf{X}^{(i)}:=\sum_{A=1}^{n_{\text {node }}^{(i)}} N_{A}(\xi) \mathbf{X}_{A}^{(i)}, \quad \mathbf{u}^{(i)}:=\sum_{A=1}^{n_{\text {node }}^{(i)}} N_{A}(\xi) \mathbf{u}_{A}^{(i)}, \quad \mathbf{V}^{(i)}:=\sum_{A=1}^{n_{\text {node }}^{(i)}} N_{A}(\xi) \mathbf{V}_{A}^{(i)}
$$

where $\mathbf{x}^{(i)}:=\mathbf{X}^{(i)}+\mathbf{u}^{(i)}$ gives the current placement of the particle $\mathbf{X}^{(i)} \in \bar{\Omega}_{h}^{(i)}$ of body $\mathcal{B}^{(i)}$, $\mathbf{X}_{A}^{(i)} \in \bar{\Omega}_{h}^{(i)}, \mathbf{u}_{A}^{(i)}$ and $\mathbf{V}_{A}^{(i)}$ are the vectors of material coordinates, displacements and material velocities, respectively, of a node $A$ of the triangulation of body $\mathcal{B}^{(i)}, N_{A}(\xi): \square \mapsto \mathbb{R}$ is the interpolation shape function for node $A, \xi \in \square$ are the isoparametric coordinates defined in the unit domain $\square$, and $n_{\text {node }}^{(i)}$ is the number of nodes used in the triangulation of body $\mathcal{B}^{(i)}$.

Consider the time interval of interest $\mathbb{I}=[0, T]$ discretized into a series of nonoverlapping sub-intervals $\mathbb{I}=\bigcup\left[t_{n}, t_{n+1}\right]$. Using the standard convention, we denote by either $(\cdot)_{n+\alpha}$ the discrete approximations at time $t_{n+\alpha}$ of the continuum variable at time $t$.

Using a mid-point time integration algorithm, the time discretization and finite element discretization of the variational form of the momentum balance equation for the dynamic case yields a discrete energy and momentum conserving time stepping algorithm, where the residual force vector of a node $A$ of body $\mathcal{B}^{(i)}$ at time $n+1$, takes the form [5],[6],[14],[24],[34],[35],[36],[37],

$$
\mathbf{g}_{A}^{(i)}\left(\mathbf{u}_{n+1}^{(i)}\right):=\frac{1}{\Delta t} \mathbf{M}_{A B}^{(i)}\left(\mathbf{V}_{B, n+1}^{(i)}-\mathbf{V}_{B, n}^{(i)}\right)+\mathbf{f}_{A, n+1 / 2}^{\mathrm{int}(i)}\left(\mathbf{u}_{n+1}^{(i)}\right)-\mathbf{f}_{A, n+1 / 2}^{e x t(i)}=\mathbf{0}
$$


where $\mathbf{M}_{A B}^{(i)}$ is the mass matrix of nodes $A$ and $B$ of body $\mathcal{B}^{(i)}, \mathbf{V}_{B, n+1}^{(i)}$ is the vector of velocities of node $B$ of body $\mathcal{B}^{(i)}$ at the time $n+1$, given by,

$$
\mathbf{V}_{B, n+1}^{(i)}=2 \mathbf{V}_{B, n+1 / 2}^{(i)}-\mathbf{V}_{B, n}^{(i)}=\frac{2}{\Delta t}\left(\mathbf{u}_{B, n+1}^{(i)}-\mathbf{u}_{B, n}^{(i)}\right)-\mathbf{V}_{B, n}^{(i)}
$$

and $\mathbf{f}_{A, n+1 / 2}^{\mathrm{int}(i)}\left(\mathbf{u}_{n+1}^{(i)}\right)$ and $\mathbf{f}_{A, n+1 / 2}^{\text {ext }(i)}$ are the nodal vectors of internal forces and external forces of node $A$ of body $\mathcal{B}^{(i)}$ at the time $n+1 / 2$, respectively.

Substituting (19) into (18), the residual force vector of a node $A$ of body $\mathcal{B}^{(i)}$ at time $n+1$, takes the form,

$$
\mathbf{g}_{A}^{(i)}\left(\mathbf{u}_{n+1}^{(i)}\right):=\frac{2}{\Delta t^{2}} \mathbf{M}_{A B}^{(i)}\left(\mathbf{u}_{B, n+1}^{(i)}-\mathbf{u}_{B, n}^{(i)}\right)-\frac{2}{\Delta t} \mathbf{M}_{A B}^{(i)} \mathbf{V}_{B, n}^{(i)}+\mathbf{f}_{A, n+1 / 2}^{\text {int }(i)}\left(\mathbf{u}_{n+1}^{(i)}\right)-\mathbf{f}_{A, n+1 / 2}^{e x t(i)}=\mathbf{0}
$$

Using an incremental iterative Newton-Raphson solution scheme, the linearization of the discrete residual force vector given by (18) yields,

$$
\mathbf{g}_{A}^{(i)}\left(\mathbf{u}_{n+1}^{(i) k+1}\right):=\mathbf{g}_{A}^{(i)}\left(\mathbf{u}_{n+1}^{(i) k}\right)+D \mathbf{g}_{A}^{(i)}\left(\mathbf{u}_{n+1}^{(i) k}\right) \cdot \Delta \mathbf{u}_{n+1}^{(i) k}=\mathbf{0}
$$

where

$$
\begin{aligned}
D \mathbf{g}_{A}^{(i)}\left(\mathbf{u}_{n+1}^{(i) k}\right) \cdot \Delta \mathbf{u}_{n+1}^{(i) k} & =\frac{2}{\Delta t^{2}} \mathbf{M}_{A B}^{(i)} \Delta \mathbf{u}_{B, n+1}^{(i) k}+D \mathbf{f}_{A, n+1 / 2}^{\operatorname{int}(i) k}\left(\mathbf{u}_{n+1}^{(i) k}\right) \cdot \Delta \mathbf{u}_{n+1}^{(i) k} \\
& =\left(\frac{2}{\Delta t^{2}} \mathbf{M}_{A B}^{(i)}+\hat{\mathbf{K}}_{A B, n+1}^{(i) k}\right) \Delta \mathbf{u}_{B, n+1}^{(i) k}:=\mathbf{K}_{A B, n+1}^{(i) k} \Delta \mathbf{u}_{B, n+1}^{(i) k}
\end{aligned}
$$

where $k$ denotes the iteration number, $\mathbf{K}_{A B, n+1}^{(i) k}$ is the $A B$ component of the tangent stiffness matrix evaluated at the iteration $k$ of the time step $n+1$, and $\Delta \mathbf{u}_{B, n+1}^{(i) k}=\mathbf{u}_{B, n+1}^{(i) k+1}-\mathbf{u}_{B, n+1}^{(i) k}$.

The space semi-discrete versions $\mathbf{L}_{h}, \mathbf{J}_{h}$ and $E_{h}$ of the linear momentum, angular momentum and total energy, respectively, take the form [3],[4],[5],[6],[34]:

$$
\begin{aligned}
\mathbf{L}_{h} & :=\sum_{i=1}^{2} \sum_{A=1}^{n_{\text {node }}^{(i)}} \mathbf{M}_{A B}^{(i)} \mathbf{V}_{B}^{(i)} \\
\mathbf{J}_{h} & :=\sum_{i=1}^{2} \mathbf{x}_{A}^{(i)} \times \mathbf{M}_{A B}^{(i)} \mathbf{V}_{B}^{(i)} \\
E_{h} & :=K_{h}+W_{h}+\Pi_{h}^{e x t}=\sum_{i=1}^{2} \frac{1}{2} \mathbf{V}_{A}^{(i)} \cdot \mathbf{M}_{A B}^{(i)} \mathbf{V}_{B}^{(i)}+W_{h}+\Pi_{h}^{e x t}
\end{aligned}
$$

where Einstein's notation has been assumed for repeated indices $A$ and $B$.

It can be shown [3],[4],[5],[6] that using this conserving time integration scheme, the full discrete version of the linear momentum $\mathbf{L}_{h}$, angular momentum $\mathbf{J}_{h}$, and total energy $E_{h}$ are conserved for a homogeneous Neumann boundary problem, characterized by no imposed boundary displacements and zero external loading, zero body forces and zero natural boundary conditions, yielding zero semi-discrete external force vector, $\mathbf{f}_{A}^{\text {ext }(i)}=\mathbf{0}$, and zero semi-discrete potential energy for the external loads, $\Pi_{h}^{\text {ext }}=0$. The discrete versión of the total energy $E_{h}$ is also conserved if the external loading is conservative. The full discrete versión of the total 
energy $E_{h}$ is also conserved if the external loading is conservative, such as, for instance, if gravitational forces are the only external forces. In this case, if the external loading is conservative, the semi-discrete versión of the potential energy for the external loads can be written as,

$$
\Pi_{h}^{e x t}=\sum_{i=1}^{2} \Pi_{h}^{e x t(i)}=-\sum_{i=1}^{2} \mathbf{f}_{A}^{e x t(i)} \cdot \mathbf{u}_{A}^{(i)}
$$

where Einstein's notation has been assumed for repeated index $A$ and the nodal vectors of external forces are constant, i.e. $\mathbf{f}_{A}^{\text {ext }(i)}=$ cte .

The conserving time integration scheme exhibits the following key properties [3],[4],[5],[6],[34]:

$$
\begin{gathered}
\sum_{i=1}^{2} \sum_{A=1}^{n_{\text {node }}^{(i)}} \mathbf{f}_{A, n+1 / 2}^{\mathrm{int}(i)}=\mathbf{0} \\
\sum_{i=1}^{2} \mathbf{x}_{A, n+1 / 2} \times \mathbf{f}_{A, n+1 / 2}^{\mathrm{int}(i)}=\mathbf{0} \\
\sum_{i=1}^{2} \mathbf{f}_{A, n+1 / 2}^{\mathrm{int}(i)} \cdot\left(\mathbf{u}_{A, n+1}^{(i)}-\mathbf{u}_{A, n}^{(i)}\right)=W_{h, n+1}-W_{h, n}
\end{gathered}
$$

where Einstein's notation has been assumed for repeated index $A$.

Furthermore, the conserving time integration scheme exhibits also the following key property, crucial to derive the conservation properties of the dynamic contact problem [3]:

$$
\sum_{i=1}^{2}\left(\mathbf{x}_{A, n+1}^{(i)}-\mathbf{x}_{A, n}^{(i)}\right) \times \mathbf{M}_{A B}^{(i)} \mathbf{V}_{B, n+1 / 2}^{(i)}=\mathbf{0}
$$

where Einstein's notation has been assumed for repeated indices $A$ and $B$.

\section{Direct elimination algorithm for contact problems}

\subsection{Introduction and notation}

Within the direct elimination algorithm for contact problems proposed in this work, the restrictions arising by the contact between the bodies are introduced through the direct elimination of the displacements of the slave nodes. From a computational implementation point of view, this direct elimination method is carried out through a number of transformations made on the global tangent operator. In order to conveniently visualize those transformations, let us introduce the following notation.

Let us consider a generic slave node $s$ which is in contact with a given master element. Let us denote by $\left\{m_{1}, \ldots, m_{\text {mnod }}\right\}$ the set of master nodes of the master element which is in contact with the slave node $s$, where mnod is the number of nodes of the master element.

Furthermore, let us introduce the following notation for the set of nodes of the slave body which are connected to the slave node $s$, including both the nodes on the slave surface and the nodes in the interior domain of the slave body, and to the set of nodes of the master surface which are connected to the master nodes $\left\{m_{1}, \ldots, m_{\text {mnod }}\right\}$, including both the nodes of the master surface and the nodes in the interior domain of the master body. 
Let us denote by $\left\{g s_{1}, \ldots, g s_{\text {gsnod }}\right\}$ the set of nodes of the slave body connected to the given slave node $s$, where gsnod is the number of nodes of this set, and let us denote by $\left\{g m_{1}, \ldots, g m_{\text {gmnod }}\right\}$ the set of nodes of the master body connected to the set of nodes $\left\{m_{1}, \ldots, m_{\text {mnod }}\right\}$, where gmnod is the number of nodes of this set.

Figure 1 shows the notation introduced for a typical slave node-master segment 2D contact problem using linear elements.

Linked to the notation introduced above for the slave and master nodes, let us introduce the following notation for the vector of displacements. Let us denote as $\mathbf{u}_{s}$ the vector of displacements of the slave node $s, \mathbf{u}_{m}$ the vector collecting the displacements of the set of nodes $\left\{m_{1}, \ldots, m_{\text {mnod }}\right\}, \mathbf{u}_{g s}$ the vector collecting the displacements of the set of nodes $\left\{g s_{1}, \ldots, g s_{g s n o d}\right\}$, and $\mathbf{u}_{g m}$ the vector collecting the displacements of the set of nodes $\left\{g m_{1}, \ldots, g m_{\text {gmnod }}\right\}, \mathbf{u}_{g 1}$ the vector collecting the vector of displacements of the remaining set of nodes of body $\mathcal{B}^{(1)}$ and $\mathbf{u}_{g 2}$ the vector collecting the vector of displacements of the remaining nodes of body $\mathcal{B}^{(2)}$.

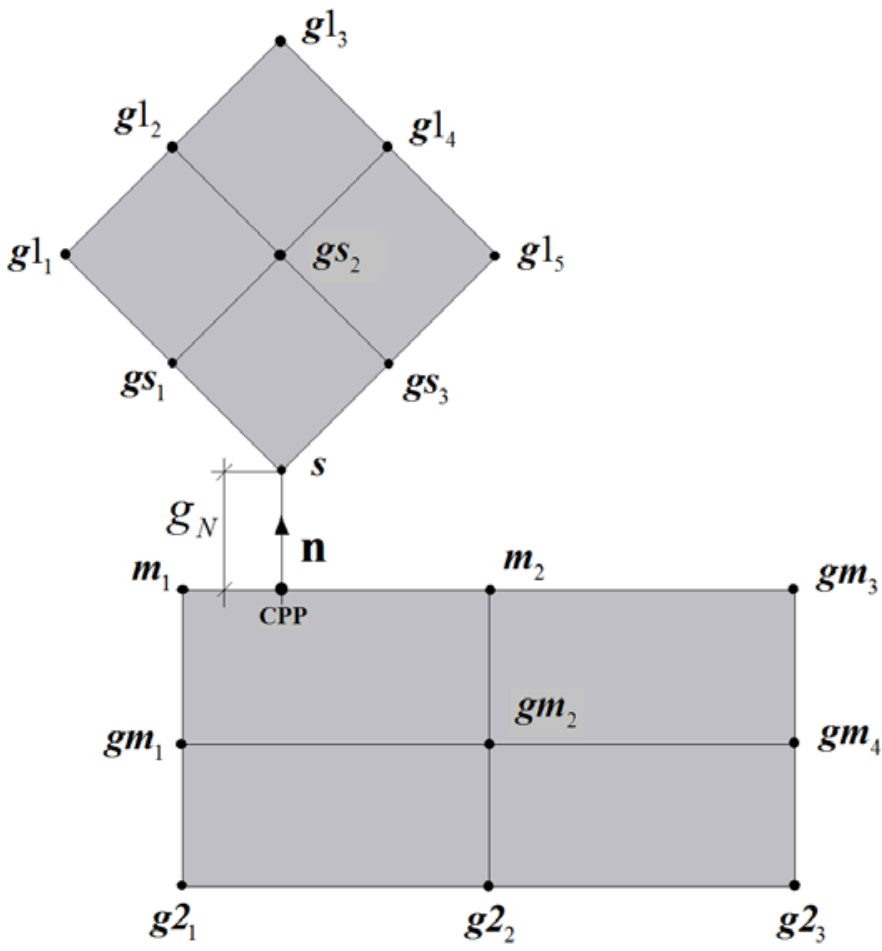

Figure 1. Definition and notation used for a slave node-to-master segment contact problem.

The vector position of an arbitrary point of a master surface element can be defined as,

$$
\mathbf{x}=\sum_{i=1}^{n_{\text {mnod }}} N_{i}(\boldsymbol{\xi}) \mathbf{x}_{m i}=\sum_{i=1}^{n_{\text {mod }}} \mathbf{N}_{i}(\boldsymbol{\xi}) \mathbf{x}_{m i}
$$

where $\xi \in \square$ are the isoparametric coordinates defined in the isoparametric unit domain $\square$, $N_{i}(\xi)$ are the interpolation shape functions of the nodes of the master element, $\mathbf{N}_{i}(\boldsymbol{\xi})=N_{i}(\boldsymbol{\xi}) \mathbf{1}$ is a diagonal matrix of shape functions.

The vector position of the closest-point-projection (CPP) of the slave node $S$ on the master element can be defined as, 


$$
\mathbf{x}_{C P P}=\sum_{i=1}^{n_{\text {mod }}} N_{i}(\bar{\xi}) \mathbf{x}_{m i}=\sum_{i=1}^{n_{\text {mood }}} \mathbf{N}_{i}(\bar{\xi}) \mathbf{x}_{m i}=\mathbf{N} \mathbf{x}_{m}
$$

where $\bar{\xi} \in \square$ are the isoparametric coordinates of the closest-point-projection defined in the isoparametric unit domain $\square$, and $\mathbf{N}=\left[\mathbf{N}_{1}(\overline{\boldsymbol{\xi}}), \ldots, \mathbf{N}_{\text {mnod }}(\overline{\boldsymbol{\xi}})\right]$ is the matrix of nodal shape functions of the master nodes evaluated at $\bar{\xi} \in \square$.

Using the notation introduced above, the semi-discrete contact normal gap $g_{N}$ can be defined as,

$$
g_{N}:=\left(\mathbf{x}_{s}-\mathbf{x}_{C P P}\right) \cdot \mathbf{n}=\left(\mathbf{x}_{s}-\sum_{i=1}^{n_{\text {mod }}} N_{i}(\bar{\xi}) \mathbf{x}_{m i}\right) \cdot \mathbf{n}=\left(\mathbf{x}_{s}-\mathbf{N} \mathbf{x}_{m}\right) \cdot \mathbf{n}
$$

where $\mathbf{x}_{s}$ is the current vector position of the slave node $S$ and $\mathbf{n}$ is the outward unit normal to the master element at the closest-point-projection of the slave node $s$.

\subsection{Contactless case}

Let us consider first a slave node $s$ which is not yet in contact with a master surface at time $n+1$. Using the notation introduced above, the residual force vectors can be written as:

$$
\begin{aligned}
\mathbf{g}_{s}\left(\mathbf{u}_{s, n+1}, \mathbf{u}_{g s, n+1}\right) & =\mathbf{0} \\
\mathbf{g}_{g s}\left(\mathbf{u}_{s, n+1}, \mathbf{u}_{g s, n+1}, \mathbf{u}_{g 1, n+1}\right) & =\mathbf{0} \\
\mathbf{g}_{g 1}\left(\mathbf{u}_{g s, n+1}, \mathbf{u}_{g 1, n+1}\right) & =\mathbf{0} \\
\mathbf{g}_{m}\left(\mathbf{u}_{m, n+1}, \mathbf{u}_{g m, n+1}\right) & =\mathbf{0} \\
\mathbf{g}_{g m}\left(\mathbf{u}_{m, n+1}, \mathbf{u}_{g m, n+1}, \mathbf{u}_{g 2, n+1}\right) & =\mathbf{0} \\
\mathbf{g}_{g 2}\left(\mathbf{u}_{g m, n+1}, \mathbf{u}_{g 2, n+1}\right) & =\mathbf{0}
\end{aligned}
$$

Using an incremental iterative Newton-Raphson solution scheme, the linearization of the above expressions (30) yields the following linearized system of equations,

$$
\begin{aligned}
\mathbf{K}_{s, s, n+1}^{k} \Delta \mathbf{u}_{s, n+1}^{k}+\mathbf{K}_{s, g s, n+1}^{k} \Delta \mathbf{u}_{g s, n+1}^{k} & =-\mathbf{g}_{s}\left(\mathbf{u}_{s, n+1}^{k}, \mathbf{u}_{g s, n+1}^{k}\right) \\
\mathbf{K}_{g s, s, n+1}^{k} \Delta \mathbf{u}_{s, n+1}^{k}+\mathbf{K}_{g s, g s, n+1}^{k} \Delta \mathbf{u}_{g s, n+1}^{k}+\mathbf{K}_{g s, g 1, n+1}^{k} \Delta \mathbf{u}_{g 1, n+1}^{k} & =-\mathbf{g}_{g s}\left(\mathbf{u}_{s, n+1}^{k}, \mathbf{u}_{g s, n+1}^{k}, \mathbf{u}_{g 1, n+1}^{k}\right) \\
\mathbf{K}_{g 1, g s, n+1}^{k} \Delta \mathbf{u}_{g s, n+1}^{k}+\mathbf{K}_{g 1, g 1, n+1}^{k} \Delta \mathbf{u}_{g 1, n+1}^{k} & =-\mathbf{g}_{g 1}\left(\mathbf{u}_{g s, n+1}^{k}, \mathbf{u}_{g 1, n+1}^{k}\right) \\
\mathbf{K}_{m, m, n+1}^{k} \Delta \mathbf{u}_{m, n+1}^{k}+\mathbf{K}_{m, g m, n+1}^{k} \Delta \mathbf{u}_{g m, n+1}^{k} & =-\mathbf{g}_{m}\left(\mathbf{u}_{m, n+1}^{k}, \mathbf{u}_{g m, n+1}^{k}\right) \\
\mathbf{K}_{g m, m, n+1}^{k} \Delta \mathbf{u}_{m, n+1}^{k}+\mathbf{K}_{g m, g m, n+1}^{k} \Delta \mathbf{u}_{g m, n+1}^{k}+\mathbf{K}_{g m, g 2, n+1}^{k} \Delta \mathbf{u}_{g 2, n+1}^{k} & =-\mathbf{g}_{g m}\left(\mathbf{u}_{m, n+1}^{k}, \mathbf{u}_{g m, n+1}^{k}, \mathbf{u}_{g 2, n+1}^{k}\right) \\
\mathbf{K}_{g 2, g m, n+1}^{k} \Delta \mathbf{u}_{g m, n+1}^{k}+\mathbf{K}_{g 2, g 2, n+1}^{k} \Delta \mathbf{u}_{g 2, n+1}^{k} & =-\mathbf{g}_{g 2}\left(\mathbf{u}_{g m, n+1}^{k}, \mathbf{u}_{g 2, n+1}^{k}\right)
\end{aligned}
$$

where $\mathbf{K}_{s, s, n+1}^{k}$ and $\mathbf{K}_{s, g s, n+1}^{k}$ are the tangent stiffness blocks corresponding to row $s$ and columns $s$ and $g s$, respectively, $\mathbf{K}_{g s, s, n+1}^{k}, \mathbf{K}_{g s, g s, n+1}^{k}$ and $\mathbf{K}_{g s, g 1, n+1}^{k}$ are the tangent stiffness blocks corresponding to row $g s$ and columns $s, g s$ and $g 1$, respectively, $\mathbf{K}_{g 1, g s, n+1}^{k}$ and $\mathbf{K}_{g 1, g 1, n+1}^{k}$ are the tangent stiffness blocks corresponding to row $g 1$ and columns $g s$ and $g 1$, 
respectively, $\mathbf{K}_{m, m, n+1}^{k}$ and $\mathbf{K}_{m, g m, n+1}^{k}$ are the tangent stiffness blocks corresponding to row $m$ and columns $m$ and $g m$, respectively, $\mathbf{K}_{g m, m, n+1}^{k}, \mathbf{K}_{g m, g m, n+1}^{k}$ and $\mathbf{K}_{g m, g 2, n+1}^{k}$ are the tangent stiffness blocks corresponding to row $\mathrm{gm}$ and columns $m, g m$ and $g 2$, respectively, and $\mathbf{K}_{g 2, g m, n+1}^{k}$ and $\mathbf{K}_{g 2, g 2, n+1}^{k}$ are the tangent stiffness blocks corresponding to row $g 2$ and columns $\mathrm{gm}$ and $\mathrm{g} 2$, respectively, all of them evaluated at the iteration $k$ of time $n+1$.

The resulting global linearized system of equations for the contactless case can be written in matrix form as,

$$
\left[\begin{array}{cccccc}
\mathbf{K}_{s, s} & \mathbf{K}_{s, g s} & \mathbf{0} & \mathbf{0} & \mathbf{0} & \mathbf{0} \\
\mathbf{K}_{g s, s} & \mathbf{K}_{g s, g s} & \mathbf{K}_{g s, g 1} & \mathbf{0} & \mathbf{0} & \mathbf{0} \\
\mathbf{0} & \mathbf{K}_{g 1, g s} & \mathbf{K}_{g 1, g 1} & \mathbf{0} & \mathbf{0} & \mathbf{0} \\
\mathbf{0} & \mathbf{0} & \mathbf{0} & \mathbf{K}_{m, m} & \mathbf{K}_{m, g m} & \mathbf{0} \\
\mathbf{0} & \mathbf{0} & \mathbf{0} & \mathbf{K}_{g m, m} & \mathbf{K}_{g m, g m} & \mathbf{K}_{g m, g 2} \\
\mathbf{0} & \mathbf{0} & \mathbf{0} & \mathbf{0} & \mathbf{K}_{g 2, g m} & \mathbf{K}_{g 2, g 2}
\end{array}\right]_{n+1}^{k}\left[\begin{array}{c}
\Delta \mathbf{u}_{s} \\
\Delta \mathbf{u}_{g s} \\
\Delta \mathbf{u}_{g 1} \\
\Delta \mathbf{u}_{m} \\
\Delta \mathbf{u}_{g m} \\
\Delta \mathbf{u}_{g 2}
\end{array}\right]_{n+1}^{k}=-\left[\begin{array}{c}
\mathbf{g}_{s} \\
\mathbf{g}_{g s} \\
\mathbf{g}_{g 1} \\
\mathbf{g}_{m} \\
\mathbf{g}_{g m} \\
\mathbf{g}_{g 2}
\end{array}\right]_{n+1}^{k}
$$

\subsection{Full stick frictional and frictionless contact cases}

Let us denote as $\mathbf{f}_{s, n+\alpha}$ the discrete contact force acting on the slave node $s$ at time $n+\alpha$, and $\mathbf{f}_{m, n+\alpha}$ the vector collecting the discrete contact forces acting on the nodes of the master element at time $n+\alpha$, where, typically, $\alpha=1 / 2$ for the dynamic case. The discrete residual force vectors can be written as:

$$
\begin{array}{r}
\mathbf{g}_{s}\left(\mathbf{u}_{s, n+1}, \mathbf{u}_{g s, n+1}\right)-\mathbf{f}_{s, n+\alpha}=\mathbf{0} \\
\mathbf{g}_{g s}\left(\mathbf{u}_{s, n+1}, \mathbf{u}_{g s, n+1}, \mathbf{u}_{g 1, n+1}\right)=\mathbf{0} \\
\mathbf{g}_{g 1}\left(\mathbf{u}_{g s, n+1}, \mathbf{u}_{g 1, n+1}\right)=\mathbf{0} \\
\mathbf{g}_{m}\left(\mathbf{u}_{m, n+1}, \mathbf{u}_{g m, n+1}\right)-\mathbf{f}_{m, n+\alpha}=\mathbf{0} \\
\mathbf{g}_{g m}\left(\mathbf{u}_{m, n+1}, \mathbf{u}_{g m, n+1}, \mathbf{u}_{g 2, n+1}\right)=\mathbf{0} \\
\mathbf{g}_{g 2}\left(\mathbf{u}_{g m, n+1}, \mathbf{u}_{g 2, n+1}\right)=\mathbf{0}
\end{array}
$$

\subsubsection{Full stick frictional contact case}

Once contact penetration is detected, the position of the slave node $S$ is subjected to the constraints arising from the full stick frictional contact condition. Note that for the full stick frictional case, once the slave node comes into contact with a master surface, the isoparametric coordinates of the closest-point-projection are time-independent, remaining constant in time while contact is active.

Using a discrete linear momentum and energy conserving time integration scheme, the mid-point velocity of the slave node $s$ is matched to the mid-point velocity of its closest-pointprojection, yielding the following expression [3]: 


$$
\mathbf{v}_{s, n+1 / 2}=\sum_{i=1}^{n_{\text {mood }}} N_{i}(\bar{\xi}) \mathbf{v}_{m i, n+1 / 2}=\sum_{i=1}^{n_{\text {mood }}} \mathbf{N}_{i}(\bar{\xi}) \mathbf{v}_{m i, n+1 / 2}=\mathbf{N} \mathbf{v}_{m, n+1 / 2}
$$

where the (time-independent) isoparametric coordinates of the closest-point-projection are computed at the mid-point configuration.

Using a mid-point rule time integration scheme, (34) yields,

$$
\mathbf{x}_{s, n+1}=\mathbf{x}_{s, n}+\mathbf{N}\left(\mathbf{x}_{m, n+1}-\mathbf{x}_{m, n}\right), \quad \Delta \mathbf{u}_{s, n+1}=\mathbf{N} \Delta \mathbf{u}_{m, n+1}
$$

Note that it is not posible to get an algorithm simultaneously satisfying discrete energy and angular momentum conservation [3]. The discrete contact constraint (34) yields a time discrete energy conservation algorithm, but the conservation of the discrete angular momentum is not satisfied.

Remark 1. Alternatively, imposing as contact constraint that the mid-point position of the slave node $s$ has to be equal to the mid-point position of its closest-point-projection, would yield a discrete momentum conservation algorithm, but then the discrete energy conservation would not be satisfied [3].

\subsubsection{Virtual contact work}

Applying the virtual work principle to the discrete contact force vectors $\mathbf{f}_{s, n+\alpha}$ and $\mathbf{f}_{m, n+\alpha}$ reads,

$$
\delta \mathbf{u}_{s} \cdot \mathbf{f}_{s, n+\alpha}+\delta \mathbf{u}_{m} \cdot \mathbf{f}_{m, n+\alpha}=0
$$

where $\delta \mathbf{u}_{s}$ and $\delta \mathbf{u}_{m}$ are virtual displacements of the slave and master element nodes, such that, taking into account that the isoparametric coordinates of the closest-point-projection remain constant, yields,

$$
\delta \mathbf{u}_{s}=\mathbf{N} \delta \mathbf{u}_{m}
$$

Substituting (37) into (36) yields,

$$
\delta \mathbf{u}_{s} \cdot \mathbf{f}_{s, n+\alpha}+\delta \mathbf{u}_{m} \cdot \mathbf{f}_{m, n+\alpha}=\delta \mathbf{u}_{m} \cdot \mathbf{N}^{T} \mathbf{f}_{s, n+\alpha}+\delta \mathbf{u}_{m} \cdot \mathbf{f}_{m, n+\alpha}=0
$$

and the discrete contact force vectors $\mathbf{f}_{s, n+\alpha}$ and $\mathbf{f}_{m, n+\alpha}$ satisfy the following expression,

$$
\mathbf{f}_{m, n+\alpha}=-\mathbf{N}^{T} \mathbf{f}_{s, n+\alpha}
$$

Using (33) $)_{1}$ and (39), the discrete contact force vectors acting on a slave node $s$ and on the master nodes $m$ at time $n+\alpha$ can be written as,

$$
\begin{array}{|l}
\mathbf{f}_{s, n+\alpha}=\mathbf{g}_{s}\left(\mathbf{u}_{s, n+1}, \mathbf{u}_{g s, n+1}\right) \\
\mathbf{f}_{m, n+\alpha}=-\mathbf{N}^{T} \mathbf{f}_{s, n+\alpha}=-\mathbf{N}^{T} \mathbf{g}_{s}\left(\mathbf{u}_{s, n+1}, \mathbf{u}_{g s, n+1}\right)
\end{array}
$$

yielding the following transformed discrete residuals, 


$$
\begin{aligned}
\mathbf{r}_{s}\left(\mathbf{u}_{s, n+1}, \mathbf{u}_{g s, n+1}\right) & :=\mathbf{0} \\
\mathbf{r}_{g s}\left(\mathbf{u}_{s, n+1}, \mathbf{u}_{g s, n+1}, \mathbf{u}_{g 1, n+1}\right) & :=\mathbf{g}_{g s}\left(\mathbf{u}_{s, n+1}, \mathbf{u}_{g s, n+1}, \mathbf{u}_{g 1, n+1}\right)=\mathbf{0} \\
\mathbf{r}_{g 1}\left(\mathbf{u}_{g s, n+1}, \mathbf{u}_{g 1, n+1}\right) & :=\mathbf{g}_{g 1}\left(\mathbf{u}_{g s, n+1}, \mathbf{u}_{g 1, n+1}\right)=\mathbf{0} \\
\mathbf{r}_{m}\left(\mathbf{u}_{m, n+1}, \mathbf{u}_{g m, n+1}, \mathbf{u}_{s, n+1}, \mathbf{u}_{g s, n+1}\right) & :=\mathbf{g}_{m}\left(\mathbf{u}_{m, n+1}, \mathbf{u}_{g m, n+1}\right)+\mathbf{N}^{T} \mathbf{g}_{s}\left(\mathbf{u}_{s, n+1}, \mathbf{u}_{g s, n+1}\right)=\mathbf{0} \\
\mathbf{r}_{g m}\left(\mathbf{u}_{m, n+1}, \mathbf{u}_{g m, n+1}, \mathbf{u}_{g 2, n+1}\right) & :=\mathbf{g}_{g m}\left(\mathbf{u}_{m, n+1}, \mathbf{u}_{g m, n+1}, \mathbf{u}_{g 2, n+1}\right)=\mathbf{0} \\
\mathbf{r}_{g 2}\left(\mathbf{u}_{g m, n+1}, \mathbf{u}_{g 2, n+1}\right) & :=\mathbf{g}_{g 2}\left(\mathbf{u}_{g m, n+1}, \mathbf{u}_{g 2, n+1}\right)=\mathbf{0}
\end{aligned}
$$

Using an incremental iterative Newton-Raphson solution scheme, taking into account that the isoparametric coordinates of th closest-point-projection remains constant, and using the notation introduced in (31) and (35), the linearization of the (non-trivial) modified residuals of interest in the above expressions (41) yields the following linearized system of equations,

$$
\begin{aligned}
& \mathbf{K}_{g s, s, n+1}^{k} \mathbf{N} \Delta \mathbf{u}_{m, n+1}^{k}+\mathbf{K}_{g s, g s, n+1}^{k} \Delta \mathbf{u}_{g s, n+1}^{k}+\mathbf{K}_{g s, g 1, n+1}^{k} \Delta \mathbf{u}_{g 1, n+1}^{k} \\
& \quad=-\mathbf{g}_{g s}\left(\mathbf{u}_{s, n+1}^{k}, \mathbf{u}_{g s, n+1}^{k}, \mathbf{u}_{g 1, n+1}^{k}\right) \\
& \left(\mathbf{K}_{m, m, n+1}^{k}+\mathbf{N}^{T} \mathbf{K}_{s, s, n+1}^{k} \mathbf{N}\right) \Delta \mathbf{u}_{m, n+1}^{k}+\mathbf{K}_{m, g m, n+1}^{k} \Delta \mathbf{u}_{g m, n+1}^{k}+\mathbf{N}^{T} \mathbf{K}_{s, g s, n+1}^{k} \Delta \mathbf{u}_{g s, n+1}^{k} \\
& \quad=-\mathbf{g}_{m}\left(\mathbf{u}_{m, n+1}^{k}, \mathbf{u}_{g m, n+1}^{k}\right)-\mathbf{N}^{T} \mathbf{g}_{s}\left(\mathbf{u}_{s, n+1}^{k}, \mathbf{u}_{g s, n+1}^{k}\right) \\
& \mathbf{K}_{g m, m, n+1}^{k} \Delta \mathbf{u}_{m, n+1}^{k}+\mathbf{K}_{g m, g m, n+1}^{k} \Delta \mathbf{u}_{g m, n+1}^{k}+\mathbf{K}_{g m, g 2, n+1}^{k} \Delta \mathbf{u}_{g 2, n+1}^{k} \\
& \quad=-\mathbf{g}_{g m}\left(\mathbf{u}_{m, n+1}^{k}, \mathbf{u}_{g m, n+1}^{k}, \mathbf{u}_{g 2, n+1}^{k}\right)
\end{aligned}
$$

The resulting modified global linearized system of equations for the full stick frictional contact case can be written in matrix form as,

$$
\left[\begin{array}{cccccc}
\beta \mathbf{1} & \mathbf{0} & \mathbf{0} & \mathbf{0} & \mathbf{0} & \mathbf{0} \\
\mathbf{0} & \mathbf{K}_{g s, g s} & \mathbf{K}_{g s, g 1} & \mathbf{K}_{g s, s} \mathbf{N} & \mathbf{0} & \mathbf{0} \\
\mathbf{0} & \mathbf{K}_{g 1, g s} & \mathbf{K}_{g 1, g 1} & \mathbf{0} & \mathbf{0} & \mathbf{0} \\
\mathbf{0} & \mathbf{N}^{T} \mathbf{K}_{s, g s} & \mathbf{0} & \mathbf{K}_{m, m}+\mathbf{N}^{T} \mathbf{K}_{s, s} \mathbf{N} & \mathbf{K}_{m, g m} & \mathbf{0} \\
\mathbf{0} & \mathbf{0} & \mathbf{0} & \mathbf{K}_{g m, m} & \mathbf{K}_{g m, g m} & \mathbf{K}_{g m, g 2} \\
\mathbf{0} & \mathbf{0} & \mathbf{0} & \mathbf{0} & \mathbf{K}_{g 2, g m} & \mathbf{K}_{g 2, g 2}
\end{array}\right]_{n+1}^{k}\left[\begin{array}{c}
\Delta \mathbf{u}_{s} \\
\Delta \mathbf{u}_{g s} \\
\Delta \mathbf{u}_{g 1} \\
\Delta \mathbf{u}_{m} \\
\Delta \mathbf{u}_{g m} \\
\Delta \mathbf{u}_{g 2}
\end{array}\right]_{n+1}^{k}=-\left[\begin{array}{c}
\mathbf{0} \\
\mathbf{g}_{g s} \\
\mathbf{g}_{g 1} \\
\mathbf{g}_{m}+\mathbf{N}^{T} \mathbf{g}_{s} \\
\mathbf{g}_{g m} \\
\mathbf{g}_{g 2}
\end{array}\right]_{n+1}^{k}
$$

The matrix $\beta_{n+1}^{k} \mathbf{1}$ is added in order to avoid the ill-conditioning (zero terms in the main diagonal) of the system, where the scalar parameter $\beta_{n+1}^{k}$ is defined as,

$$
\beta_{n+1}^{k}=\frac{1}{n_{\mathrm{dim}}} \mathbf{1}: \mathbf{K}_{s, s, n+1}^{k}=\frac{1}{n_{\mathrm{dim}}} \operatorname{tr}\left[\mathbf{K}_{s, s, n+1}^{k}\right]
$$

where $\operatorname{tr}[\cdot]$ denotes the trace operator and $n_{\text {dim }}$ is the number of dimensions of the problem. 
Remark 3. Note that block-symmetry of the resulting global tangent stiffness matrix for the full stick friction case is preserved.

\subsubsection{Update of slave and master displacements and contact status}

Once the resulting incremental iterative problem has been solved, the slave and master displacements are updated according to the following expressions.

Using a discrete linear momentum and energy conserving algorithm, the update of the master and slave and displacements takes the form,

$$
\begin{aligned}
\mathbf{u}_{m, n+1}^{k+1} & =\mathbf{u}_{m, n+1}^{k}+\Delta \mathbf{u}_{m, n+1}^{k} \\
\mathbf{u}_{s, n+1}^{k+1} & =\mathbf{u}_{s, n+1}^{k}+\Delta \mathbf{u}_{s, n+1}^{k}=\mathbf{u}_{s, n+1}^{k}+\mathbf{N} \Delta \mathbf{u}_{m, n+1}^{k}
\end{aligned}
$$

Once the displacements of the slave and master nodes have been updated, the nodal velocities of the slave and master nodes are updated using (19).

The contact status at time $n+\alpha$ has to be verified, checking out if the contact is still active or not. The contact will be still active if the contact normal force $f_{N_{n+\alpha}}:=\mathbf{f}_{s, n+\alpha} \cdot \mathbf{n}_{n+\alpha}$ satisfies the following condition:

$$
f_{N_{n+\alpha}}:=\mathbf{f}_{s, n+\alpha} \cdot \mathbf{n}_{n+\alpha}=\mathbf{g}_{s}\left(\mathbf{u}_{s, n+1}, \mathbf{u}_{g s, n+1}\right) \cdot \mathbf{n}_{n+\alpha} \geq 0
$$

Otherwise, contact is lost and the contact status for the slave node $S$ has to be deactivated for the next time step.

\subsubsection{Frictionless contact case}

Once contact penetration is detected, the position of the slave node $s$ is subjected to the constraints arising from the frictionless contact condition. Note that, contrary to the full stick frictional case, for the frictionless case, the isoparametric coordinates of the closest-pointprojection are not constant in time. For the sake of concreteness, only 3D dynamic frictionless contact cases will be presented here, being straightforward to particularize the formulation for 2D cases.

A discrete linear momentum, angular momentum and energy conserving algorithm is obtained, imposing that the normal component of the slave node at the mid-point configuration has to be equal to the normal component of the velocity of its closest-point-projection at the mid-point configuration, yielding,

$$
\mathbf{v}_{s, n+1 / 2} \cdot \mathbf{n}_{n+1 / 2}=\sum_{i=1}^{n_{\text {mood }}} N_{i}\left(\overline{\boldsymbol{\xi}}_{n+1 / 2}\right) \mathbf{v}_{m i, n+1 / 2} \cdot \mathbf{n}_{n+1 / 2}=\mathbf{N}_{n+1 / 2} \mathbf{v}_{m, n+1 / 2} \cdot \mathbf{n}_{n+1 / 2}
$$

where $\mathbf{n}_{n+1 / 2}$ is the outward unit normal to the closest-point-projection at the configuration at time $n+1 / 2$.

Using a mid-point time integration scheme, the normal velocity constraint given by (46) yields the following kinematic constraint, 


$$
\mathbf{x}_{s, n+1} \cdot \mathbf{n}_{n+1 / 2}=\left(\mathbf{x}_{s, n}+\mathbf{N}_{n+1 / 2}\left(\mathbf{x}_{m, n+1}-\mathbf{x}_{m, n}\right)\right) \cdot \mathbf{n}_{n+1 / 2}
$$

Then, the mid-point velocity of the slave node $s$ at time $n+1 / 2$, and the current placement and current increment of displacement of the slave node $s$ at time $n+1$ can be written as,

$$
\begin{aligned}
\mathbf{v}_{s, n+1 / 2} & =\mathbf{N}_{n+1 / 2} \mathbf{v}_{m, n+1 / 2}+\dot{\bar{\xi}}_{n+1 / 2} \boldsymbol{\tau}_{\xi, n+1 / 2}+\dot{\bar{\eta}}_{n+1 / 2} \tau_{\eta, n+1 / 2} \\
\mathbf{x}_{s, n+1} & =\mathbf{x}_{s, n}+\mathbf{N}_{n+1 / 2}\left(\mathbf{x}_{m, n+1}-\mathbf{x}_{m, n}\right)+\Delta \bar{\xi}_{n+1} \tau_{\xi, n+1 / 2}+\Delta \bar{\eta}_{n+1} \tau_{\eta, n+1 / 2} \\
\Delta \mathbf{u}_{s, n+1} & =\mathbf{N}_{n+1 / 2} \Delta \mathbf{u}_{m, n+1}+\Delta \bar{\xi}_{n+1} \boldsymbol{\tau}_{\xi, n+1 / 2}+\Delta \bar{\eta}_{n+1} \tau_{\eta, n+1 / 2}
\end{aligned}
$$

where $\left(\Delta \bar{\xi}_{n+1}, \Delta \bar{\eta}_{n+1}\right)$ are the contravariant components of the isoparametric coordinates of the incremental slip of the closest-point-projection on the covariant basis vectors at the mid-point configuration $\left(\tau_{\xi, n+1 / 2}, \tau_{\eta, n+1 / 2}\right)$. Note that the vectors defining the covariant tangent basis do not need to be neither unit vectors, nor orthogonal.

\subsubsection{Virtual contact work}

Applying the virtual work principle to the discrete contact force vectors $\mathbf{f}_{s, n+\alpha}$ and $\mathbf{f}_{m, n+\alpha}$ reads,

$$
\delta \mathbf{u}_{s} \cdot \mathbf{f}_{s, n+\alpha}+\delta \mathbf{u}_{m} \cdot \mathbf{f}_{m, n+\alpha}=0
$$

where $\delta \mathbf{u}_{s}$ and $\delta \mathbf{u}_{m}$ are virtual displacements of the slave node and master element nodes, respectively, such that,

$$
\delta \mathbf{u}_{s}=\mathbf{N}_{n+1 / 2} \delta \mathbf{u}_{m}+\tau_{\xi, n+1 / 2} \delta \bar{\xi}_{n+1}+\tau_{\eta, n+1 / 2} \delta \bar{\eta}_{n+1}=\mathbf{N}_{n+1 / 2} \delta \mathbf{u}_{m}+\mathbf{T}_{n+1 / 2} \delta \bar{\xi}_{n+1}
$$

where $\delta \overline{\boldsymbol{\xi}}_{n+1}$ and $\mathbf{T}_{n+1 / 2}$ have been defined, conveniently expanded, as,

$$
\delta \overline{\boldsymbol{\xi}}_{n+1}=\left[\delta \bar{\xi}_{n+1}, \delta \bar{\eta}_{n+1}, 0\right]^{T}, \quad \mathbf{T}_{n+1 / 2}=\left[\boldsymbol{\tau}_{\xi, n+1 / 2}, \tau_{\eta, n+1 / 2}, \mathbf{0}\right]
$$

Substituting (50) into (49), and taking into account that for a frictionless case, $\tau_{\xi, n+1 / 2} \cdot \mathbf{f}_{s, n+\alpha}=0$ and $\tau_{\eta, n+1 / 2} \cdot \mathbf{f}_{s, n+\alpha}=0$, yields,

$$
\delta \mathbf{u}_{m} \cdot \mathbf{N}_{n+1 / 2}^{T} \mathbf{f}_{s, n+\alpha}+\delta \mathbf{u}_{m} \cdot \mathbf{f}_{m, n+\alpha}=0
$$

and the discrete contact force vectors $\mathbf{f}_{s, n+\alpha}$ and $\mathbf{f}_{m, n+\alpha}$ have to satisfy the following expressions,

$$
\begin{aligned}
\mathbf{f}_{m, n+\alpha} & =-\mathbf{N}_{n+1 / 2}^{T} \mathbf{f}_{s, n+\alpha} \\
\mathbf{0} & =\mathbf{T}_{n+1 / 2}^{T} \mathbf{f}_{s, n+\alpha}
\end{aligned}
$$

Using (33) $)_{1}$ and (53) and, the discrete contact force vectors acting on a slave node $s$ and on the master nodes $m$ at time $n+\alpha$ can be written as, 


$$
\begin{aligned}
\mathbf{f}_{s, n+\alpha} & =\mathbf{g}_{s}\left(\mathbf{u}_{s, n+1}, \mathbf{u}_{g s, n+1}\right) \\
\mathbf{f}_{m, n+\alpha} & =-\mathbf{N}_{n+1 / 2}^{T} \mathbf{f}_{s, n+\alpha}=-\mathbf{N}_{n+1 / 2}^{T} \mathbf{g}_{s}\left(\mathbf{u}_{s, n+1}, \mathbf{u}_{g s, n+1}\right) \\
\mathbf{0} & =\mathbf{T}_{n+1 / 2}^{T} \mathbf{f}_{s, n+\alpha}=\mathbf{T}_{n+1 / 2}^{T} \mathbf{g}_{s}\left(\mathbf{u}_{s, n+1}, \mathbf{u}_{g s, n+1}\right)
\end{aligned}
$$

yielding the following modified residuals,

$$
\begin{aligned}
\mathbf{r}_{s}\left(\mathbf{u}_{s, n+1}, \mathbf{u}_{g s, n+1}\right) & :=\mathbf{T}_{n+1 / 2}^{T} \mathbf{g}_{s}\left(\mathbf{u}_{s, n+1}, \mathbf{u}_{g s, n+1}\right)=\mathbf{0} \\
\mathbf{r}_{g s}\left(\mathbf{u}_{s, n+1}, \mathbf{u}_{g s, n+1}, \mathbf{u}_{g 1, n+1}\right) & :=\mathbf{g}_{g s}\left(\mathbf{u}_{s, n+1}, \mathbf{u}_{g s, n+1}, \mathbf{u}_{g 1, n+1}\right)=\mathbf{0} \\
\mathbf{r}_{g 1}\left(\mathbf{u}_{g s, n+1}, \mathbf{u}_{g 1, n+1}\right) & :=\mathbf{g}_{g 1}\left(\mathbf{u}_{g s, n+1}, \mathbf{u}_{g 1, n+1}\right)=\mathbf{0} \\
\mathbf{r}_{m}\left(\mathbf{u}_{m, n+1}, \mathbf{u}_{g m, n+1}, \mathbf{u}_{s, n+1}, \mathbf{u}_{g s, n+1}\right) & :=\mathbf{g}_{m}\left(\mathbf{u}_{m, n+1}, \mathbf{u}_{g m, n+1}\right)+\mathbf{N}_{n+1 / 2}^{T} \mathbf{g}_{s}\left(\mathbf{u}_{s, n+1}, \mathbf{u}_{g s, n+1}\right)=\mathbf{0} \\
\mathbf{r}_{g m}\left(\mathbf{u}_{m, n+1}, \mathbf{u}_{g m, n+1}, \mathbf{u}_{g 2, n+1}\right) & :=\mathbf{g}_{g m}\left(\mathbf{u}_{m, n+1}, \mathbf{u}_{g m, n+1}, \mathbf{u}_{g 2, n+1}\right)=\mathbf{0} \\
\mathbf{r}_{g 2}\left(\mathbf{u}_{g m, n+1}, \mathbf{u}_{g 2, n+1}\right) & :=\mathbf{g}_{g 2}\left(\mathbf{u}_{g m, n+1}, \mathbf{u}_{g 2, n+1}\right)=\mathbf{0}
\end{aligned}
$$

Using an incremental iterative Newton-Raphson solution scheme, taking into account the variation of the closest-point-projection, the linearization of the modified residuals in the above expressions takes the form:

$$
\begin{aligned}
\mathbf{r}_{s} & \left(\mathbf{u}_{s, n+1}^{k+1}, \mathbf{u}_{g s, n+1}^{k+1}\right):=\mathbf{T}_{n+1 / 2}^{T k} \mathbf{g}_{s}\left(\mathbf{u}_{s, n+1}^{k}, \mathbf{u}_{g s, n+1}^{k}\right) \\
& +\mathbf{T}_{n+1 / 2}^{T k} D \mathbf{g}_{s}\left(\mathbf{u}_{s, n+1}^{k}, \mathbf{u}_{g s, n+1}^{k}\right) \cdot \Delta \mathbf{u}_{s, n+1}^{k}+\mathbf{T}_{n+1 / 2}^{T k} D \mathbf{g}_{s}\left(\mathbf{u}_{s, n+1}^{k}, \mathbf{u}_{g s, n+1}^{k}\right) \cdot \Delta \mathbf{u}_{g s, n+1}^{k} \\
& +\Delta \mathbf{T}_{n+1 / 2}^{T k} \mathbf{g}_{s}\left(\mathbf{u}_{s, n+1}^{k}, \mathbf{u}_{g s, n+1}^{k}\right)=\mathbf{0} \\
\mathbf{r}_{g s} & \left(\mathbf{u}_{s, n+1}^{k+1}, \mathbf{u}_{g s, n+1}^{k+1}, \mathbf{u}_{g 1, n+1}^{k+1}\right):=\mathbf{g}_{g s}\left(\mathbf{u}_{s, n+1}^{k}, \mathbf{u}_{g s, n+1}^{k}, \mathbf{u}_{g 1, n+1}^{k}\right) \\
& +D \mathbf{g}_{g s}\left(\mathbf{u}_{s, n+1}^{k}, \mathbf{u}_{g s, n+1}^{k}, \mathbf{u}_{g 1, n+1}^{k}\right) \cdot \Delta \mathbf{u}_{s, n+1}^{k}+D \mathbf{g}_{g s}\left(\mathbf{u}_{s, n+1}^{k}, \mathbf{u}_{g s, n+1}^{k}, \mathbf{u}_{g 1, n+1}^{k}\right) \cdot \Delta \mathbf{u}_{g s, n+1}^{k} \\
& +D \mathbf{g}_{g s}\left(\mathbf{u}_{s, n+1}^{k}, \mathbf{u}_{g s, n+1}^{k}, \mathbf{u}_{g 1, n+1}^{k}\right) \cdot \Delta \mathbf{u}_{g 1, n+1}^{k}=\mathbf{0} \\
\mathbf{r}_{m} & \left(\mathbf{u}_{m, n+1}^{k+1}, \mathbf{u}_{g m, n+1}^{k+1}, \mathbf{u}_{s, n+1}^{k+1}, \mathbf{u}_{g s, n+1}^{k+1}\right):=\mathbf{g}_{m}\left(\mathbf{u}_{m, n+1}^{k}, \mathbf{u}_{g m, n+1}^{k}\right)+\mathbf{N}_{n+1 / 2}^{T k} \mathbf{g}_{s}\left(\mathbf{u}_{s, n+1}^{k}, \mathbf{u}_{g s, n+1}^{k}\right) \\
& +D \mathbf{g}_{m}\left(\mathbf{u}_{m, n+1}^{k}, \mathbf{u}_{g m, n+1}^{k}\right) \cdot \Delta \mathbf{u}_{m, n+1}^{k}+D \mathbf{g}_{m}\left(\mathbf{u}_{m, n+1}^{k}, \mathbf{u}_{g m, n+1}^{k}\right) \cdot \Delta \mathbf{u}_{g m, n+1}^{k} \\
& +\mathbf{N}_{n+1 / 2}^{T k} D \mathbf{g}_{s}\left(\mathbf{u}_{s, n+1}^{k}, \mathbf{u}_{g s, n+1}^{k}\right) \cdot \Delta \mathbf{u}_{s, n+1}^{k}+\mathbf{N}_{n+1 / 2}^{T k} D \mathbf{g}_{s}\left(\mathbf{u}_{s, n+1}^{k}, \mathbf{u}_{g s, n+1}^{k}\right) \cdot \Delta \mathbf{u}_{g s, n+1}^{k} \\
& +\Delta \mathbf{N}_{n+1 / 2}^{T k} \mathbf{g}_{s}\left(\mathbf{u}_{s, n+1}^{k}, \mathbf{u}_{g s, n+1}^{k}\right)=\mathbf{0} \\
\mathbf{r}_{g m} & \left(\mathbf{u}_{m, n+1}^{k+1}, \mathbf{u}_{g m, n+1}^{k+1}, \mathbf{u}_{g 2, n+1}^{k+1}\right):=\mathbf{g}_{g m}\left(\mathbf{u}_{m, n+1}^{k}, \mathbf{u}_{g m, n+1}^{k}, \mathbf{u}_{g 2, n+1}^{k}\right) \\
& +D \mathbf{g}_{g m}\left(\mathbf{u}_{m, n+1}^{k}, \mathbf{u}_{g m, n+1}^{k}, \mathbf{u}_{g 2, n+1}^{k}\right) \cdot \Delta \mathbf{u}_{m, n+1}^{k}+D \mathbf{g}_{g m}\left(\mathbf{u}_{m, n+1}^{k}, \mathbf{u}_{g m, n+1}^{k}, \mathbf{u}_{g 2, n+1}^{k}\right) \cdot \Delta \mathbf{u}_{g m, n+1}^{k} \\
& +D \mathbf{g}_{g m}\left(\mathbf{u}_{m, n+1}^{k}, \mathbf{u}_{g m, n+1}^{k}, \mathbf{u}_{g 2, n+1}^{k}\right) \cdot \Delta \mathbf{u}_{g 2, n+1}^{k}=\mathbf{0}
\end{aligned}
$$

The resulting global linearized system of equations for the frictionless contact case can be written in matrix form as, 
$\left[\begin{array}{cccccc}\mathbf{T}^{T} \mathbf{K}_{s, s} \mathbf{T}+\overline{\mathbf{A}}+\beta \mathbf{1}_{001} & \mathbf{T}^{T} \mathbf{K}_{s, g s} & \mathbf{0} & \mathbf{T}^{T} \mathbf{K}_{s, s} \mathbf{N}+\mathbf{A}^{T} & \mathbf{0} & \mathbf{0} \\ \mathbf{K}_{g s, s} \mathbf{T} & \mathbf{K}_{g s, g s} & \mathbf{K}_{g s, g 1} & \mathbf{K}_{g s, s} \mathbf{N} & \mathbf{0} & \mathbf{0} \\ \mathbf{0} & \mathbf{K}_{g 1, g s} & \mathbf{K}_{g 1, g 1} & \mathbf{0} & \mathbf{0} & \mathbf{0} \\ \mathbf{N}^{T} \mathbf{K}_{s, s} \mathbf{T}+\mathbf{A} & \mathbf{N}^{T} \mathbf{K}_{s, g s} & \mathbf{0} & \mathbf{K}_{m, m}+\mathbf{N}^{T} \mathbf{K}_{s, s} \mathbf{N} & \mathbf{K}_{m, g m} & \mathbf{0} \\ \mathbf{0} & \mathbf{0} & \mathbf{0} & \mathbf{K}_{g m, m} & \mathbf{K}_{g m, g m} & \mathbf{K}_{g m, g 2} \\ \mathbf{0} & \mathbf{0} & \mathbf{0} & \mathbf{0} & \mathbf{K}_{g 2, g m} & \mathbf{K}_{g 2, g 2}\end{array}\right]_{n+1}^{k}\left[\begin{array}{c}\Delta \overline{\boldsymbol{\xi}} \\ \Delta \mathbf{u}_{g s} \\ \Delta \mathbf{u}_{g 1} \\ \Delta \mathbf{u}_{m} \\ \Delta \mathbf{u}_{g m} \\ \Delta \mathbf{u}_{g 2}\end{array}\right]_{n+1}^{k}=-\left[\begin{array}{c}\mathbf{T}^{T} \mathbf{g}_{s} \\ \mathbf{g}_{g s} \\ \mathbf{g}_{g 1} \\ \mathbf{g}_{m}+\mathbf{N}^{T} \mathbf{g}_{s} \\ \mathbf{g}_{g m} \\ \mathbf{g}_{g 2}\end{array}\right]_{n+1}^{k}$

where note that matrices $\mathbf{T}, \mathbf{A}, \overline{\mathbf{A}}, \mathbf{N}$ have to be evaluated at the configuration $n+1 / 2$, as indicated in (57), and $\mathbf{1}_{001}$ is a $n_{\mathrm{dim}} \times n_{\mathrm{dim}}$ matrix with zero entries everywhere, except a 1 entry in the diagonal position $\left(n_{\mathrm{dim}}, n_{\mathrm{dim}}\right)$. The matrix $\beta_{n+1}^{k} \mathbf{1}_{001}$ is added in order to avoid the illconditioning (zero terms in the main diagonal) of the system, where the scalar parameter $\beta_{n+1}^{k}$ is defined as,

$$
\beta_{n+1}^{k}=\frac{1}{n_{\mathrm{dim}}} \mathbf{1}: \mathbf{K}_{s, s, n+1}^{k}=\frac{1}{n_{\mathrm{dim}}} \operatorname{tr}\left[\mathbf{K}_{s, s, n+1}^{k}\right]
$$

where $\operatorname{tr}[\cdot]$ denotes the trace operator and $n_{\text {dim }}$ is the number of dimensions of the problem. Matrices $\mathbf{A}, \overline{\mathbf{A}}$ arise from the variation of the covariant tangent basis vectors $\tau_{\xi, n+\alpha}^{k}$ and $\tau_{\eta, n+\alpha}^{k}$, and the variation of $\mathbf{N}_{n+\alpha}^{k}$ in (57), respectively. For convenience, those variations are given in the Appendix 1. Note that for linear elements the condition $\overline{\mathbf{A}}=\mathbf{0}$ holds.

Note that, following this procedure, the number of equations of the system is kept constant. Once convergence has been achieved, the increment of displacements $\Delta \mathbf{u}_{s, n+1}$ is computed in terms of $\Delta \mathbf{u}_{m, n+1}$ and $\Delta \overline{\boldsymbol{\xi}}_{n+1}$ using (49) . $_{3}$

Remark 4. Note that, using the formulation presented here, block-symmetry of the resulting global tangent stiffness matrix for the frictionless case is also maintained, in contrast to what happened in the formulation shown in Di Capua \& Agelet de Saracibar (2015) [9].

\subsubsection{Update of slave and master displacements and contact status}

The displacements of the master nodes at the iteration $k+1$ of time $n+1$ are updated according to the following expression,

$$
\mathbf{u}_{m, n+1}^{k+1}=\mathbf{u}_{m, n+1}^{k}+\Delta \mathbf{u}_{m, n+1}^{k}
$$

The displacements of the slave nodes at the iteration $k+1$ of time $n+1$ are updated according to the following procedure:

Step 1. Compute the current displacements and coordinates of the slave node at the iteration $k+1$ of time $n+1$, using the isoparametric coordinates $\bar{\xi}_{n+1 / 2}^{k}$ of the closest-pointprojection at the iteration $k$,

$$
\begin{aligned}
& \overline{\mathbf{u}}_{s, n+1}^{k+1}=\mathbf{u}_{s, n+1}^{k}+\mathbf{N}_{n+1 / 2}^{k} \Delta \mathbf{u}_{m, n+1}^{k}+\Delta \bar{\xi}_{n+1}^{k} \tau_{\xi, n+1 / 2}^{k}+\Delta \bar{\eta}_{n+1}^{k} \tau_{\eta, n+1 / 2}^{k} \\
& \overline{\mathbf{x}}_{s, n+1}^{k+1}=\mathbf{X}_{s}+\overline{\mathbf{u}}_{s, n+1}^{k+1}
\end{aligned}
$$


Step 2. Update the isoparametric coordinates $\overline{\boldsymbol{\xi}}_{n+1 / 2}^{k+1}$ of the closest-point-projection of the slave node at the iteration $k+1$ using the coordinates $\overline{\mathbf{x}}_{s, n+1 / 2}^{k+1}$ of the slave node defined as,

$$
\overline{\mathbf{x}}_{s, n+1 / 2}^{k+1}=\frac{1}{2}\left(\overline{\mathbf{x}}_{s, n+1}^{k+1}+\mathbf{x}_{s, n}\right)
$$

Step 3. Update the current displacements of the slave node at the iteration $k+1$ of time $n+1$ according to the following expression:

$$
\mathbf{u}_{s, n+1}^{k+1}=\overline{\mathbf{x}}_{s, n+1}^{k+1}-\Delta g_{N, n+1 / 2}^{k+1} \mathbf{n}_{n+1 / 2}^{k+1}-\mathbf{X}_{s}
$$

where,

$$
\Delta g_{N, n+1 / 2}^{k+1}=\left(\overline{\mathbf{x}}_{s, n+1}^{k+1}-\mathbf{x}_{s, n}-\mathbf{N}_{n+1 / 2}^{k+1}\left(\mathbf{x}_{m, n+1}^{k+1}-\mathbf{x}_{m, n}\right)\right) \cdot \mathbf{n}_{n+1 / 2}^{k+1}
$$

such that, it is ensured that the discrete frictionless contact kinematic constraint given by (48) and (49) is satisfied, and the discrete linear momentum, angular momentum and energy are conserved.

Once the displacements of the slave and master nodes have been updated, the nodal velocities of the slave and master nodes are updated using (19).

Once the displacements have been updated, the contact status has to be checked out in order to decide if it has to be keep as active or if it has to be deactivated for the next step. The contact will be still active if the contact normal force $f_{N_{n+\alpha}}:=\mathbf{f}_{s, n+\alpha} \cdot \mathbf{n}_{n+\alpha}$ satisfies the following condition:

$$
f_{N_{n+\alpha}}:=\mathbf{f}_{s, n+\alpha} \cdot \mathbf{n}_{n+\alpha}=\mathbf{g}_{s}\left(\mathbf{u}_{s, n+1}, \mathbf{u}_{g s, n+1}\right) \cdot \mathbf{n}_{n+\alpha} \geq 0
$$

Otherwise, contact is lost and the contact status for the slave node $s$ has to be deactivated for the next time step.

\section{Conservation properties of the proposed algorithm for the dynamic contact case}

\subsection{Full stick frictional contact case}

The proposed contact algorithm exhibits the following conservation properties for the full stick frictional contact case: conservation of the discrete linear momentum for a homogeneous Neumann problem, and conservation of the discrete total energy for constant gravitational loading. It must be pointed out that for the full stick frictional contact case the discrete angular momentum is not conserved.

\subsubsection{Conservation of the discrete linear momentum}

Assuming a homogeneous Neumann problem, characterized by no imposed boundary displacements and zero external forces, such that, 


$$
\mathbf{f}_{A, n+1 / 2}^{\text {ext }(i)}=\mathbf{0}
$$

the following expression holds for the discrete linear momentum,

$$
\begin{aligned}
\mathbf{L}_{h, n+1}-\mathbf{L}_{h, n} & =\sum_{i=1}^{2} \sum_{A=1}^{n_{\text {node }}^{(i)}} \mathbf{M}_{A B}^{(i)}\left(\mathbf{V}_{B, n+1}^{(i)}-\mathbf{V}_{B, n}^{(i)}\right) \\
& =-\Delta t \sum_{i=1}^{2} \sum_{A=1}^{n_{\text {node }}^{(i)}} \mathbf{f}_{A, n+1 / 2}^{\mathrm{int}(i)}+\Delta t \sum_{s=1}^{s n o d}\left(\mathbf{f}_{s, n+1 / 2}+\sum_{i=1}^{\operatorname{mnod}(s)} \mathbf{f}_{m i, n+1 / 2}\right) \\
& =\Delta t \sum_{s=1}^{\text {snod }}\left(1-\sum_{i=1}^{m n o d(s)} N_{i}(\bar{\xi})\right) \mathbf{g}_{s}\left(\mathbf{u}_{s, n+1}, \mathbf{u}_{g s, n+1}\right) \\
& =\mathbf{0}
\end{aligned}
$$

where Einstein's notation has been assumed for repeated index $B$ and the following expressions have been used,

$$
\begin{gathered}
\sum_{i=1}^{2} \sum_{A=1}^{n_{\text {node }}^{(i)}} \mathbf{f}_{A, n+1 / 2}^{\mathrm{int}(i)}=\mathbf{0} \\
\mathbf{f}_{s, n+1 / 2}=\mathbf{g}_{s}\left(\mathbf{u}_{s, n+1}, \mathbf{u}_{g s, n+1}\right), \quad \mathbf{f}_{m i, n+1 / 2}=-N_{i}(\bar{\xi}) \mathbf{g}_{s}\left(\mathbf{u}_{s, n+1}, \mathbf{u}_{g s, n+1}\right)
\end{gathered}
$$

Then, for a homogeneous Neumann problem, characterized by no imposed boundary displacements and zero external loading, the discrete linear momentum is conserved, yielding,

$$
\mathbf{L}_{h, n+1}=\mathbf{L}_{h, n}
$$

\subsubsection{Conservation of the discrete total energy}

Assuming no imposed boundary displacements and (constant) gravitational forces as only external loading, such that,

$$
\mathbf{f}_{A, n}^{\text {ext }(i)}=\mathbf{f}_{A, n+1 / 2}^{\text {ext }(i)}=\mathbf{f}_{A, n+1}^{\text {ext }(i)}=c t e
$$

the following expressions hold for the discrete external potential energy, discrete elastic strain energy and discrete kinetic energy,

$$
\begin{aligned}
\prod_{h, n+1}^{e x t}-\prod_{h, n}^{e x t} & =-\sum_{i=1}^{2} \mathbf{f}_{A, n+1 / 2}^{e x t(i)} \cdot \Delta \mathbf{u}_{A, n+1}^{(i)} \\
W_{h, n+1}-W_{h, n} & =\sum_{i=1}^{2} \mathbf{f}_{A, n+1 / 2}^{\mathrm{int}(i)} \cdot \Delta \mathbf{u}_{A, n+1}^{(i)} \\
K_{h, n+1}-K_{h, n} & =\sum_{i=1}^{2} \frac{1}{2}\left(\mathbf{V}_{A, n+1}^{(i)} \cdot \mathbf{M}_{A B}^{(i)} \mathbf{V}_{B, n+1}^{(i)}-\mathbf{V}_{A, n}^{(i)} \cdot \mathbf{M}_{A B}^{(i)} \mathbf{V}_{B, n}^{(i)}\right) \\
& =\sum_{i=1}^{2} \mathbf{V}_{A, n+1 / 2}^{(i)} \cdot \mathbf{M}_{A B}^{(i)}\left(\mathbf{V}_{B, n+1}^{(i)}-\mathbf{V}_{B, n}^{(i)}\right) \\
& =\sum_{i=1}^{2} \mathbf{f}_{A, n+1 / 2}^{e x t(i)} \cdot \Delta \mathbf{u}_{A, n+1}^{(i)}-\sum_{i=1}^{2} \mathbf{f}_{A, n+1 / 2}^{\mathrm{int}(i)} \cdot \Delta \mathbf{u}_{A, n+1}^{(i)} \\
& +\sum_{s=1}^{s n o d}\left(\mathbf{f}_{s, n+1 / 2} \cdot \Delta \mathbf{u}_{s, n+1}+\mathbf{f}_{m, n+1 / 2} \cdot \Delta \mathbf{u}_{m, n+1}\right) \\
& =-\left(\Pi_{h, n+1}^{e x t}-\Pi_{h, n}^{e x t}\right)-\left(W_{h, n+1}-W_{h, n}\right)
\end{aligned}
$$


where Einstein's notation has been assumed for repeated indices $A$ and $B$ and the following expression has been used,

$$
\begin{aligned}
0 & =\sum_{s=1}^{s n o d}\left(\mathbf{f}_{s, n+1 / 2} \cdot \Delta \mathbf{u}_{s, n+1}+\mathbf{f}_{m, n+1 / 2} \cdot \Delta \mathbf{u}_{m, n+1}\right) \\
& =\sum_{s=1}^{s n o d}\left(\mathbf{g}_{s}\left(\mathbf{u}_{s, n+1}, \mathbf{u}_{g s, n+1}\right) \cdot \mathbf{N} \Delta \mathbf{u}_{m, n+1}-\mathbf{N}^{T} \mathbf{g}_{s}\left(\mathbf{u}_{s, n+1}, \mathbf{u}_{g s, n+1}\right) \cdot \Delta \mathbf{u}_{m, n+1}\right)
\end{aligned}
$$

Then, for no imposed boundary displacements and constant gravitational forces as only external loading, the discrete total energy is conserved, yielding,

$$
E_{h, n+1}=K_{h, n+1}+W_{h, n+1}+\Pi_{h, n+1}^{e x t}=K_{h, n}+W_{h, n}+\Pi_{h, n}^{e x t}=E_{h, n}
$$

\subsection{Frictionless contact case}

The proposed contact algorithm exhibits the following conservation properties for the frictionless contact case: conservation of the discrete linear momentum for a homogeneous Neumann problem, conservation of the discrete angular momentum for a homogeneous Neumann problem, and conservation of the discrete total energy for constant gravitational forces as only external loading.

\subsubsection{Conservation of the discrete linear momentum}

Assuming a homogeneous Neumann problem, characterized by no imposed boundary displacements and zero external loading, such that,

$$
\mathbf{f}_{A, n+1 / 2}^{e x t(i)}=\mathbf{0}
$$

and following a similar development to the one used for the full stick frictional case, it can be shown that the discrete linear momentum is conserved, yielding,

$$
\mathbf{L}_{h, n+1}=\mathbf{L}_{h, n}
$$

\subsubsection{Conservation of the discrete angular momentum}

Assuming a homogeneous Neumann problem, characterized by no imposed boundary displacements and zero external loading, such that,

$$
\mathbf{f}_{A, n+1 / 2}^{e x t(i)}=\mathbf{0}
$$

the following expression holds for the discrete angular momentum, 


$$
\begin{aligned}
\mathbf{J}_{h, n+1}-\mathbf{J}_{h, n} & =\sum_{i=1}^{2} \mathbf{x}_{A, n+1}^{(i)} \times \mathbf{M}_{A B}^{(i)} \mathbf{V}_{B, n+1}^{(i)}-\sum_{i=1}^{2} \mathbf{x}_{A, n}^{(i)} \times \mathbf{M}_{A B}^{(i)} \mathbf{V}_{B, n}^{(i)} \\
& =\sum_{i=1}^{2} \mathbf{x}_{A, n+1 / 2}^{(i)} \times \mathbf{M}_{A B}^{(i)}\left(\mathbf{V}_{B, n+1}^{(i)}-\mathbf{V}_{B, n}^{(i)}\right)-\sum_{i=1}^{2}\left(\mathbf{x}_{A, n+1}^{(i)}-\mathbf{x}_{A, n}^{(i)}\right) \times \mathbf{M}_{A B}^{(i)} \mathbf{V}_{B, n+1 / 2}^{(i)} \\
& =-\Delta t \sum_{i=1}^{2} \mathbf{x}_{A, n+1 / 2}^{(i)} \times \mathbf{f}_{A, n+1 / 2}^{\text {int }(i)} \\
& +\Delta t \sum_{s=1}^{\text {snod }}\left(\mathbf{x}_{s, n+1 / 2} \times \mathbf{f}_{s, n+1 / 2}+\sum_{i=1}^{\operatorname{mnod}(s)} \mathbf{x}_{m i, n+1 / 2} \times \mathbf{f}_{m i, n+1 / 2}\right) \\
& =-\Delta t \sum_{s=1}^{\text {snod }}\left(\mathbf{x}_{s, n+1 / 2}-\sum_{i=1}^{m n o d(s)} N_{i}\left(\bar{\xi}_{n+1 / 2}\right) \mathbf{x}_{m i, n+1 / 2}\right) \times f_{N_{n+1 / 2}} \mathbf{n}_{n+1 / 2} \\
& =-\Delta t \sum_{s=1}^{\text {snod }} f_{N_{n+1 / 2}} g_{N_{n+1 / 2}} \mathbf{n}_{n+1 / 2} \times \mathbf{n}_{n+1 / 2} \\
& =\mathbf{0}
\end{aligned}
$$

where the following expressions have been used,

$$
\begin{gathered}
\sum_{i=1}^{2}\left(\mathbf{x}_{A, n+1}^{(i)}-\mathbf{x}_{A, n}^{(i)}\right) \times \mathbf{M}_{A B}^{(i)} \mathbf{V}_{B, n+1 / 2}^{(i)}=\mathbf{0} \\
\mathbf{f}_{s, n+1 / 2}=f_{N_{n+1 / 2}} \mathbf{n}_{n+1 / 2}, \quad \mathbf{f}_{m i, n+1 / 2}=-N_{i}\left(\bar{\xi}_{n+1 / 2}\right) f_{N_{n+1 / 2}} \mathbf{n}_{n+1 / 2}=-\mathbf{N}_{n+1 / 2} f_{N_{n+1 / 2}} \mathbf{n}_{n+1 / 2} \\
g_{N_{n+1 / 2}} \mathbf{n}_{n+1 / 2}=\mathbf{x}_{s, n+1 / 2}-\sum_{i=1}^{m n o d(s)} N_{i}\left(\bar{\xi}_{n+1 / 2}\right) \mathbf{x}_{m i, n+1 / 2}=\mathbf{x}_{s, n+1 / 2}-\mathbf{N}_{n+1 / 2} \mathbf{x}_{m, n+1 / 2}
\end{gathered}
$$

where

$$
g_{N_{n+1 / 2}}=\left(\mathbf{x}_{s, n+1 / 2}-\mathbf{N}_{n+1 / 2} \mathbf{x}_{m, n+1 / 2}\right) \cdot \mathbf{n}_{n+1 / 2}
$$

and Einstein's notation has been assumed for repeated indices $A$ and $B$.

Then, for a homogeneous Neumann problem, characterized by no imposed boundary displacements and zero external loading, the discrete angular momentum is conserved, yielding,

$$
\mathbf{J}_{h, n+1}=\mathbf{J}_{h, n}
$$

\subsubsection{Conservation of the discrete total energy}

Assuming no imposed boundary displacements and (constant) gravitational force as only external loading, i.e. such that,

$$
\mathbf{f}_{A, n}^{\text {ext }(i)}=\mathbf{f}_{A, n+1 / 2}^{\text {ext }(i)}=\mathbf{f}_{A, n+1}^{\text {ext }(i)}=c t e
$$

the following expressions hold for the discrete external potential energy, discrete elastic strain energy and discrete kinetic energy, 


$$
\begin{aligned}
\prod_{h, n+1}^{e x t}-\Pi_{h, n}^{e x t} & =-\sum_{i=1}^{2} \mathbf{f}_{A, n+1 / 2}^{e x t(i)} \cdot \Delta \mathbf{u}_{A, n+1}^{(i)} \\
W_{h, n+1}-W_{h, n} & =\sum_{i=1}^{2} \mathbf{f}_{A, n+1 / 2}^{\mathrm{int}(i)} \cdot \Delta \mathbf{u}_{A, n+1}^{(i)} \\
K_{h, n+1}-K_{h, n} & =\sum_{i=1}^{2} \frac{1}{2}\left(\mathbf{V}_{A, n+1}^{(i)} \cdot \mathbf{M}_{A B}^{(i)} \mathbf{V}_{B, n+1}^{(i)}-\mathbf{V}_{A, n}^{(i)} \cdot \mathbf{M}_{A B}^{(i)} \mathbf{V}_{B, n}^{(i)}\right) \\
& =\sum_{i=1}^{2} \mathbf{V}_{A, n+1 / 2}^{(i)} \cdot \mathbf{M}_{A B}^{(i)}\left(\mathbf{V}_{B, n+1}^{(i)}-\mathbf{V}_{B, n}^{(i)}\right) \\
& =\sum_{i=1}^{2} \mathbf{f}_{A, n+1 / 2}^{e x t(i)} \cdot \Delta \mathbf{u}_{A, n+1}^{(i)}-\sum_{i=1}^{2} \mathbf{f}_{A, n+1 / 2}^{\mathrm{int}(i)} \cdot \Delta \mathbf{u}_{A, n+1}^{(i)} \\
& +\sum_{s=1}^{s n o d}\left(\mathbf{f}_{s, n+1 / 2} \cdot \Delta \mathbf{u}_{s, n+1}+\mathbf{f}_{m, n+1 / 2} \cdot \Delta \mathbf{u}_{m, n+1}\right) \\
& =-\left(\Pi_{h, n+1}^{e x t}-\Pi_{h, n}^{e x t}\right)-\left(W_{h, n+1}-W_{h, n}\right)
\end{aligned}
$$

where Einstein's notation has been assumed for repeated indices $A$ and $B$ and the following expression has been used,

$$
\begin{aligned}
0 & =\sum_{s=1}^{\text {snod }}\left(\mathbf{f}_{s, n+1 / 2} \cdot \Delta \mathbf{u}_{s, n+1}+\mathbf{f}_{m, n+1 / 2} \cdot \Delta \mathbf{u}_{m, n+1}\right) \\
& =\sum_{s=1}^{s n o d}\left(\Delta \mathbf{u}_{s, n+1}-\mathbf{N}_{n+1 / 2} \Delta \mathbf{u}_{m, n+1}\right) \cdot f_{N_{n+1 / 2}} \mathbf{n}_{n+1 / 2}
\end{aligned}
$$

Then, for no imposed prescribed displacements and constant gravitational force as only external loading, the discrete total energy is conserved, yielding,

$$
E_{h, n+1}=K_{h, n+1}+W_{h, n+1}+\Pi_{h, n+1}^{e x t}=K_{h, n}+W_{h, n}+\Pi_{h, n}^{e x t}=E_{h, n}
$$

\section{Numerical examples}

In this section a selection of representative dynamic contact numerical examples, that illustrate the performance and conservation properties inherited by the contact formulation proposed, is shown. Two frictionless and one full stick friction dynamic contact numerical examples have been chosen.

The first example deals with the numerical simulation of the dynamic impact of three aligned quasi-rigid cylinders sliding on a frictionless rigid surface. Here the goal is to show the conservation of the discrete total linear momentum, discrete total angular momentum and discrete total energy exhibited by the contact algorithm proposed.

The second example deals with the numerical simulation of the dynamic impact of two elastic hollow cylinders sliding on a frictionless rigid surface. Here, once again, the goal is to show that the proposed formulation exhibits the conservation of the discrete total linear momentum, discrete total angular momentum and discrete total energy.

Finally, the last example deals with the numerical simulation of an elastic ball bouncing on a rigid surface. Full stick friction conditions are considered here. Now the goal is to show that the proposed contact formulation exhibits the conservation of the discrete total energy.

The numerical simulations have been performed using an enhanced version of the finite element program RamSeries [33] developed by COMPASS, a spin-off company of CIMNE. 


\subsection{Impact of three aligned quasi-rigid cylinders sliding on a frictionless rigid surface}

This example leads with the impact of three aligned quasi-rigid cylinders sliding on a rigid surface. Frictionless contact conditions between each one of the cylinders and between each one of the cyinders and the sliding surface have been considered. The radius of the cylinders is 0.1 m. Elastic Saint-Venant material models with Young's elastic modulus E=2.1E+09 $\mathrm{Pa}$ and Poisson's coefficient $v=0.3$, have been considered. The mass density of the material is 2500 $\mathrm{Kg} / \mathrm{m}^{3}$. Cylinder 1 has an initial velocity of $1.0 \mathrm{~m} / \mathrm{sec}$ in the $\mathrm{x}$-direction.

The cylinders have been discretized using standard Galerkin P1 linear displacement tetrahedral finite elements. Figure 2 shows the geometry, initial conditions and finite element meshes of the three cylinders. Time increments of $0.002 \mathrm{sec}$ have been considered.

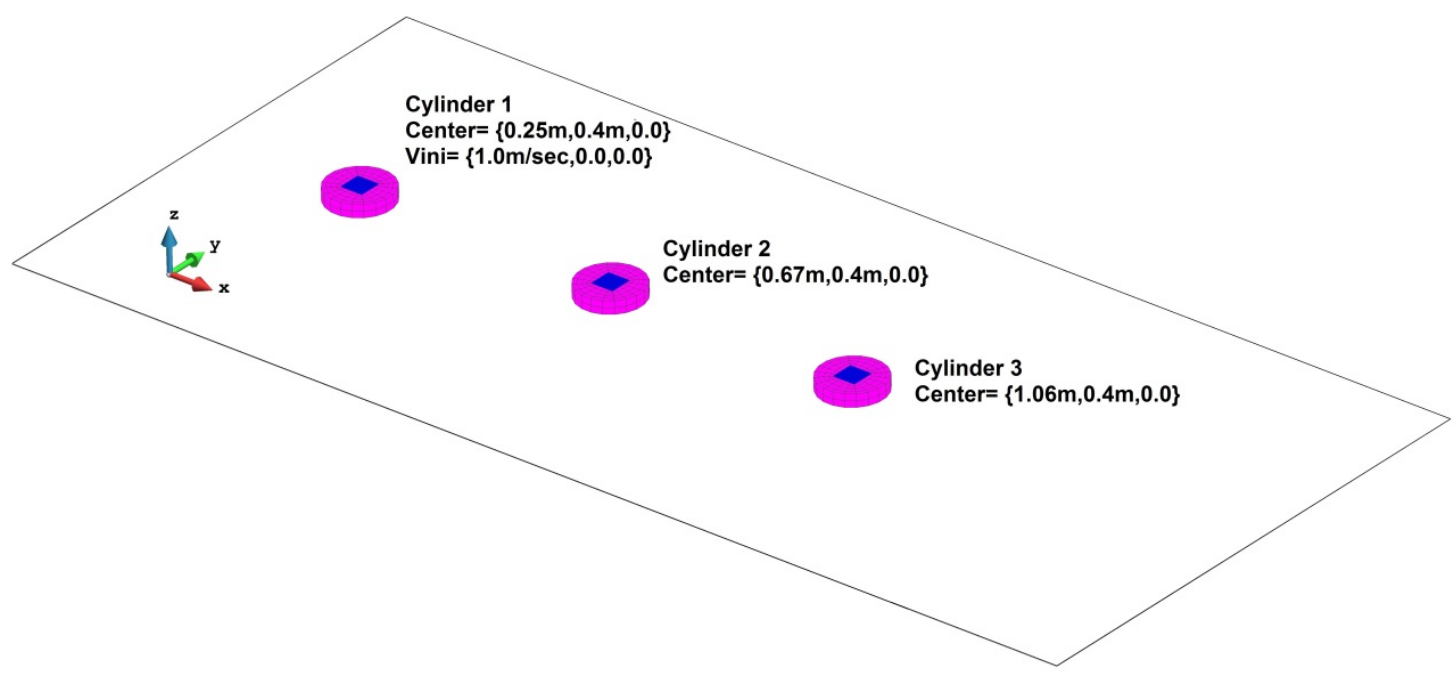

Figure 2. Impact of three aligned quasi-rigid cylinders sliding on a frictionless rigid surface. Geometry, mesh and initial conditions

Figure 3 shows the evolution of the positions of the three cylinders at different time steps. It can be shown that when the first cylinders impact on the second one, the first one stops and transfers its full velocity to the second one. This effect is repeated when the second cylinder impacts the third one. This phenomen, which can be usually observed in the pool game when aligned balls impact, is due to the conservation of the total linear momentum and total energy conservation.The proposed contact model is able to conserve the total linear momentum, total angular momentum and the total energy, as it is shown in Figures 4, 5 and 6, respectively. 


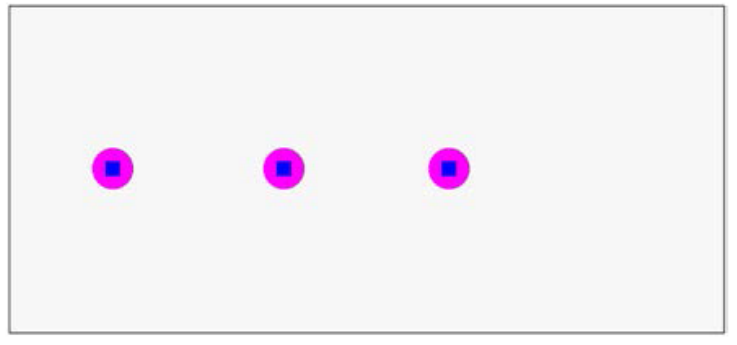

$\mathrm{t}=0.0 \mathrm{sec}$

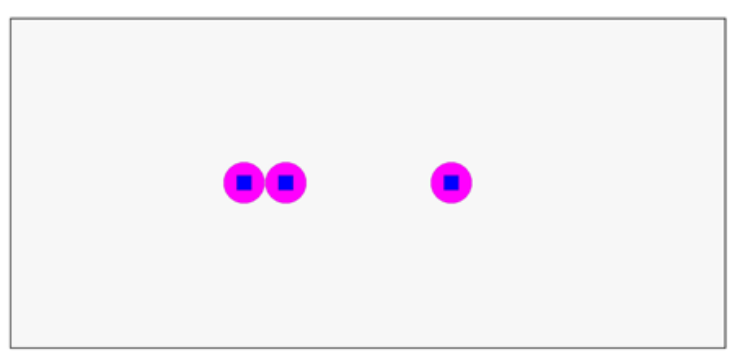

$t=0.31 \mathrm{sec}$

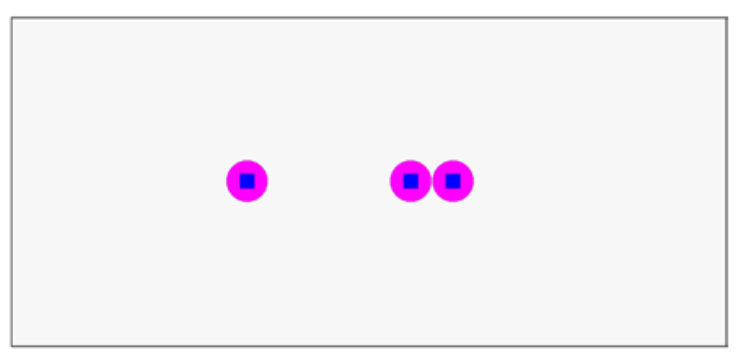

$t=0.62 \mathrm{sec}$

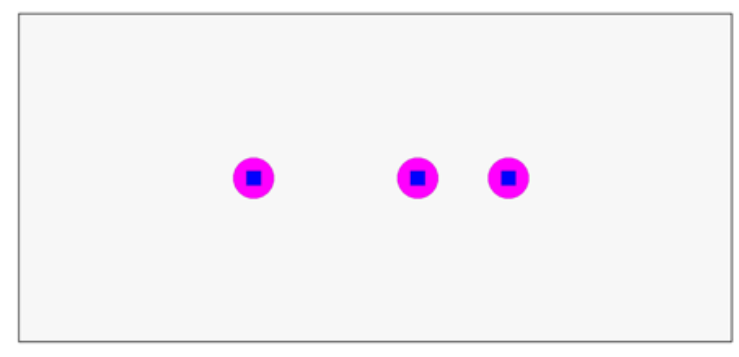

$\mathrm{t}=0.74 \mathrm{sec}$

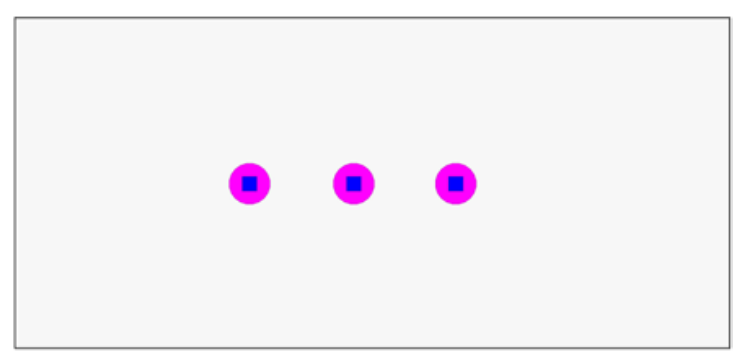

$\mathrm{t}=0.47 \mathrm{sec}$

$\mathrm{t}=0.16 \mathrm{sec}$

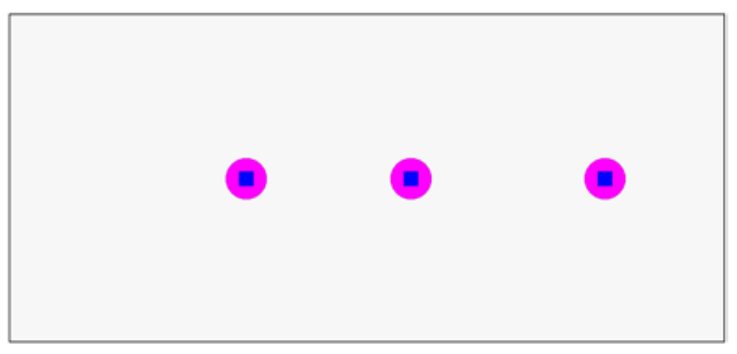

$\mathrm{t}=1.0 \mathrm{sec}$ 


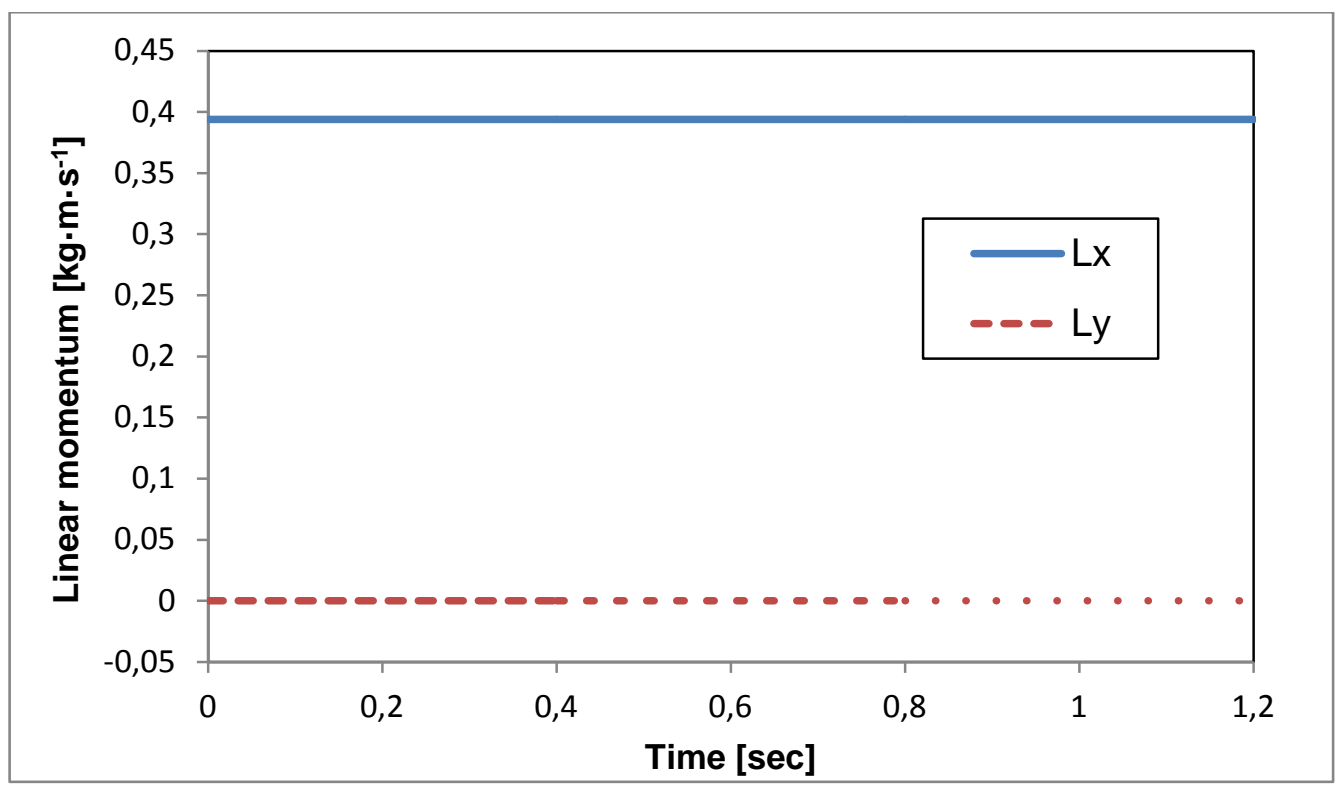

Figure 4. Impact of three aligned quasi-rigid cylinders sliding on a frictionless rigid surface. Linear momentum evolution.

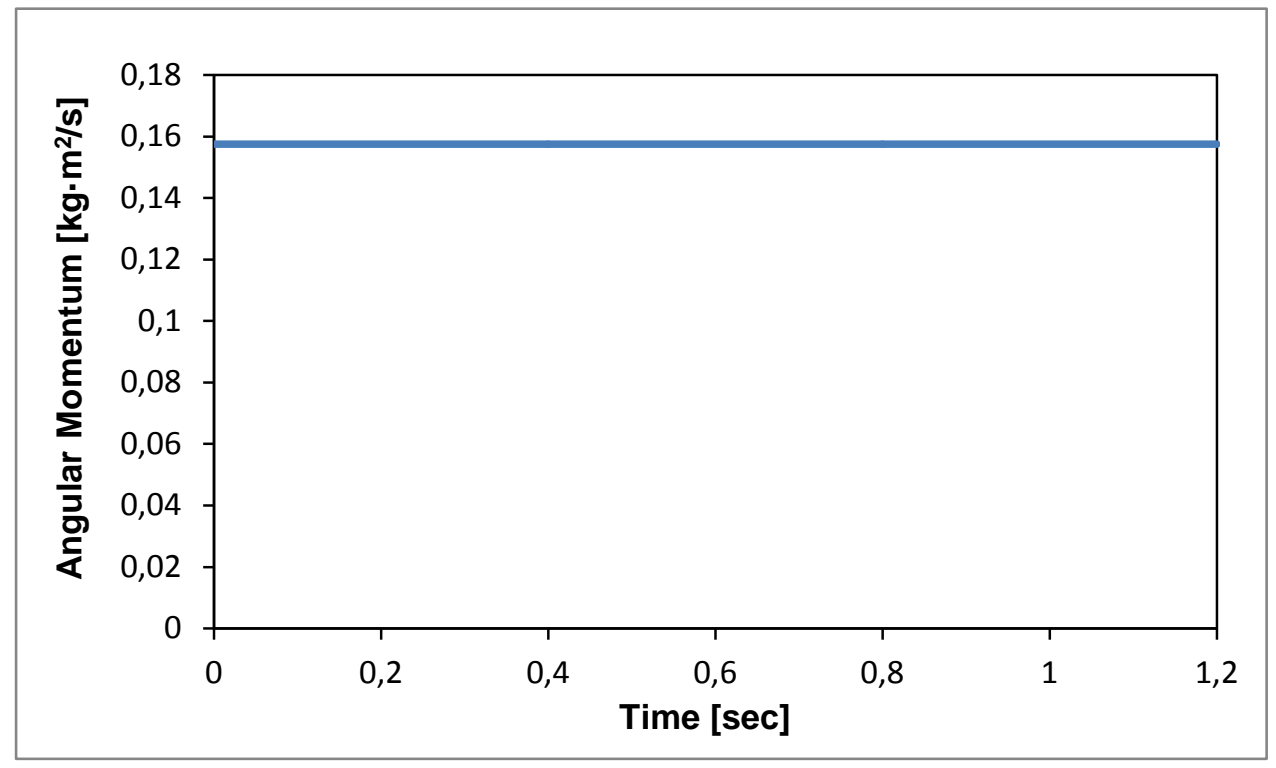

Figure 5. Impact of three aligned quasi-rigid cylinders sliding on a frictionless rigid surface. Angular momentum evolution. 


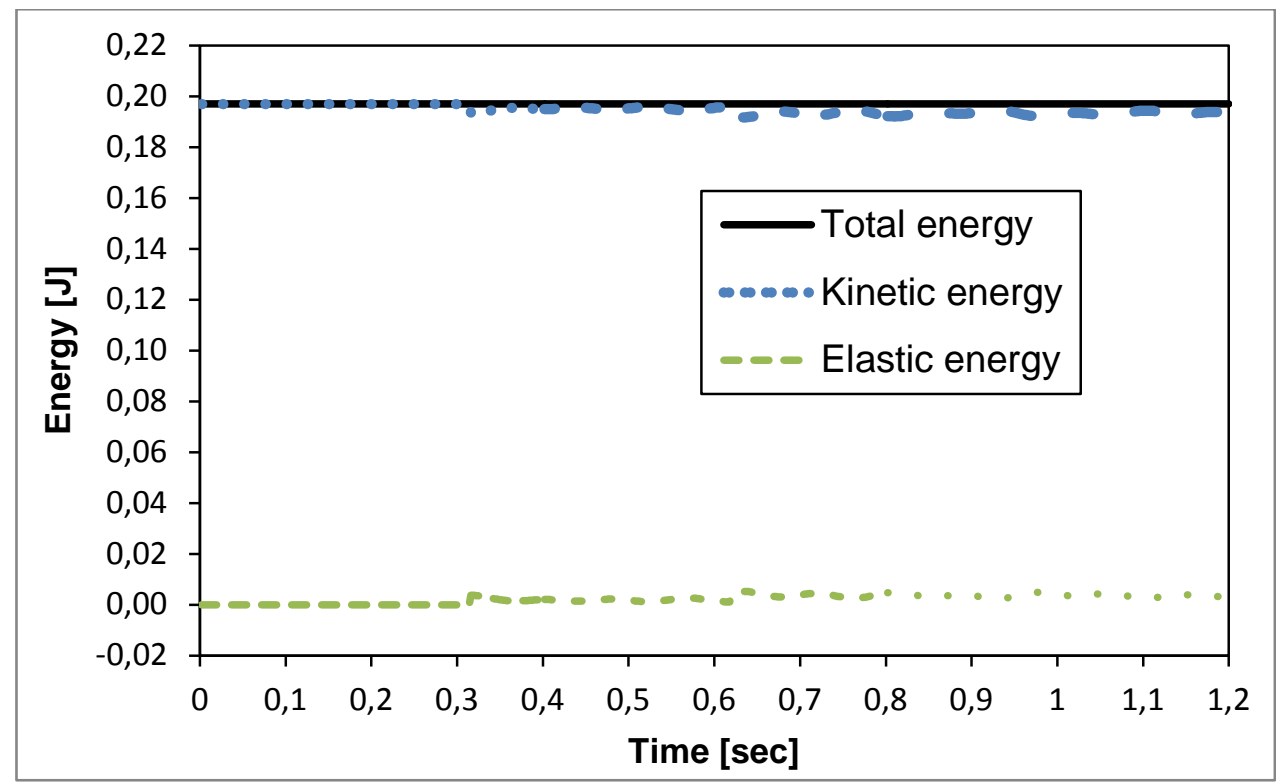

Figure 6. Impact of three aligned quasi-rigid cylinders sliding on a frictionless rigid surface. Evolution of the different energies.

\subsection{Impact of two elastic hollow cylinders sliding on a frictionless rigid surface}

This example leads with the impact of two elastic hollow cylinders sliding on a rigid surface. Frictionless contact conditions between the three cylinders and between each one of the cyinders and the sliding surface have been considered. The radius of the cylinders is $0.1 \mathrm{~m}$. Elastic Saint-Venant material models with Young's elastic modulus E=2.1E+05 $\mathrm{Pa}$ and Poisson's coefficient $v=0.3$, have been considered. The mass density of the material is 2500 $\mathrm{Kg} / \mathrm{m}^{3}$. Cylinder 1 has an initial velocity of $1.0 \mathrm{~m} / \mathrm{sec}$ in the $\mathrm{x}$-direction.

The cylinders have been discretized using standard Galerkin P1 linear displacement tetrahedral finite elements. Figure 7 shows the geometry, initial conditions and finite element meshes of the two cylinders. Time increments of 2.0e-4 sec have been considered.

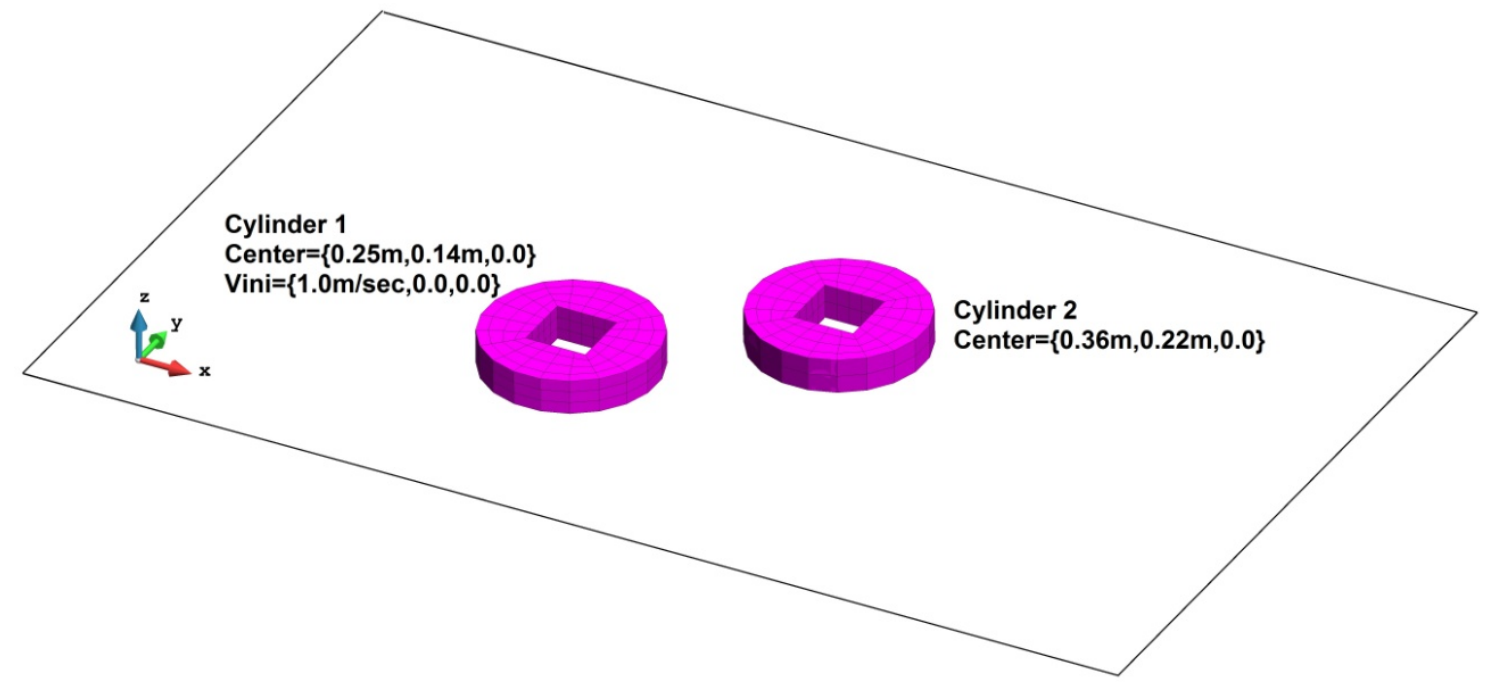

Figure 7. Impact of two aligned elastic hollow cylinders sliding on a frictionless rigid surface. Geometry, mesh and initial conditions. 


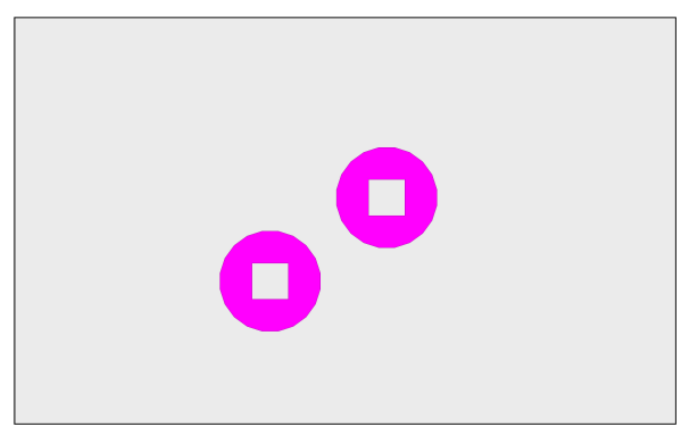

$\mathrm{t}=0.000 \mathrm{sec}$

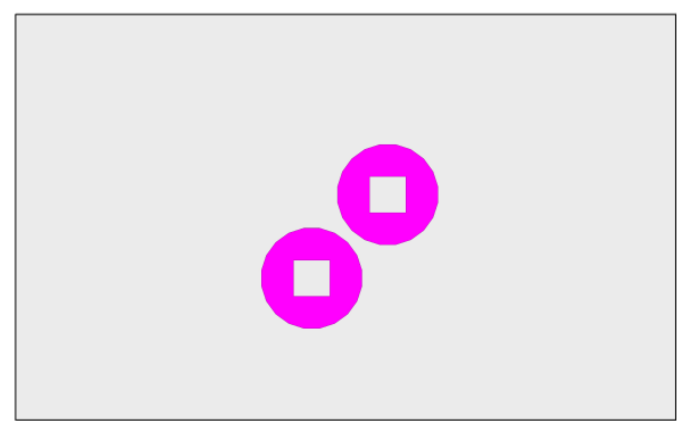

$\mathrm{t}=0.040 \mathrm{sec}$

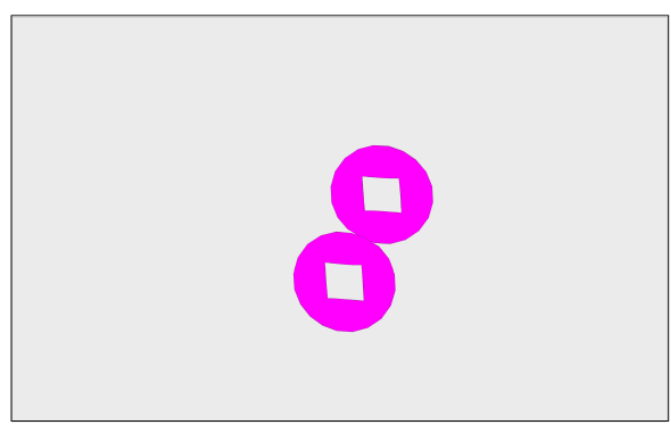

$\mathrm{t}=0.080 \mathrm{sec}$

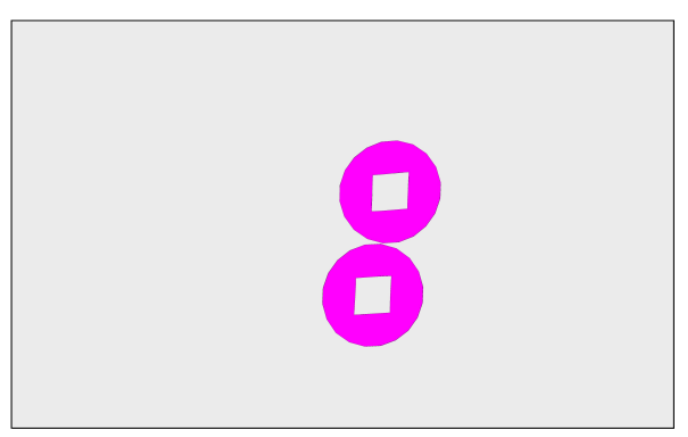

$\mathrm{t}=0.108 \mathrm{sec}$

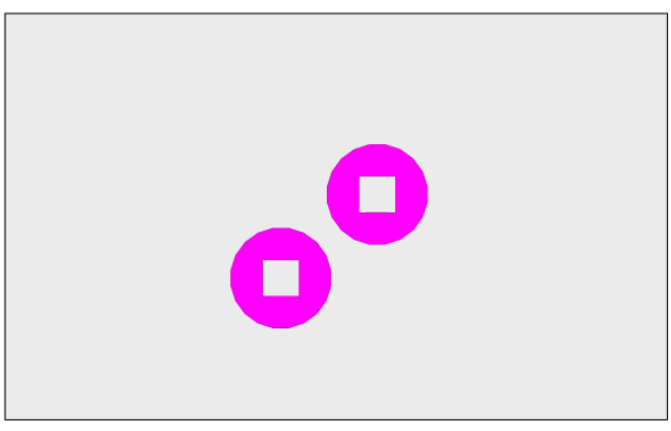

$\mathrm{t}=0.020 \mathrm{sec}$

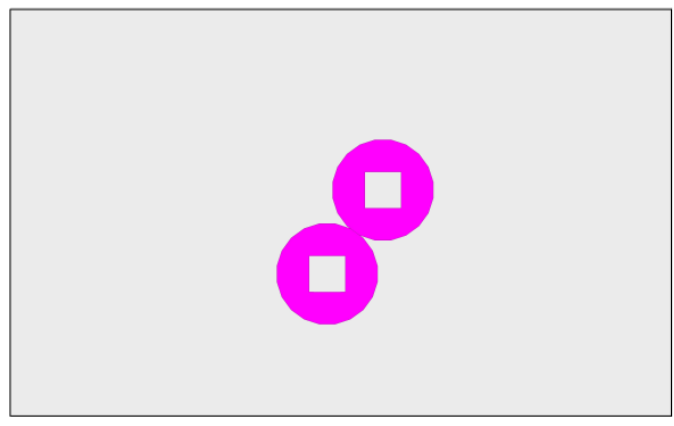

$t=0.060 \mathrm{sec}$

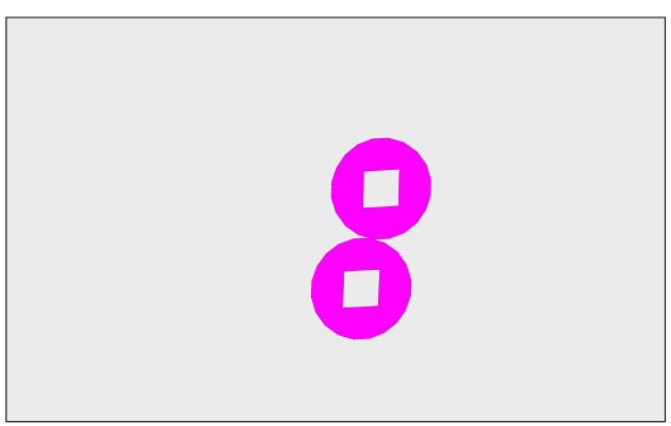

$\mathrm{t}=0.104 \mathrm{sec}$

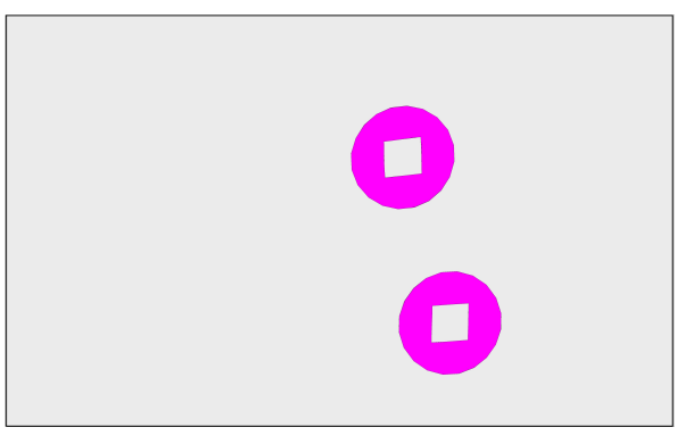

$\mathrm{t}=0.200 \mathrm{sec}$

Figure 8. Impact of two aligned elastic hollow cylinders sliding on a frictionless rigid surface. Configurations at different time steps. 
Figure 8 shows the evolution of the positions of the three cylinders at different time steps. Despite the fact that frictionless conditions have been considered, Figure 8 shows that the cylinders rotate around their axes. This is an undesirable effect due to the finite element discretization of the cylinders. Unless an extremely fine mesh is used, the unit normal to the master surface at the closest-point-projection does not pass through the axis of the cylinder, leading to a moment when the cylinders come into contact. A similar effect can be found in the results shown in [3]. Despite those undesirable effects, the proposed contact model is able to conserve the total linear momentum, total angular momentum and the total energy, as it is shown on Figures 9, 10 and 11, respectively.

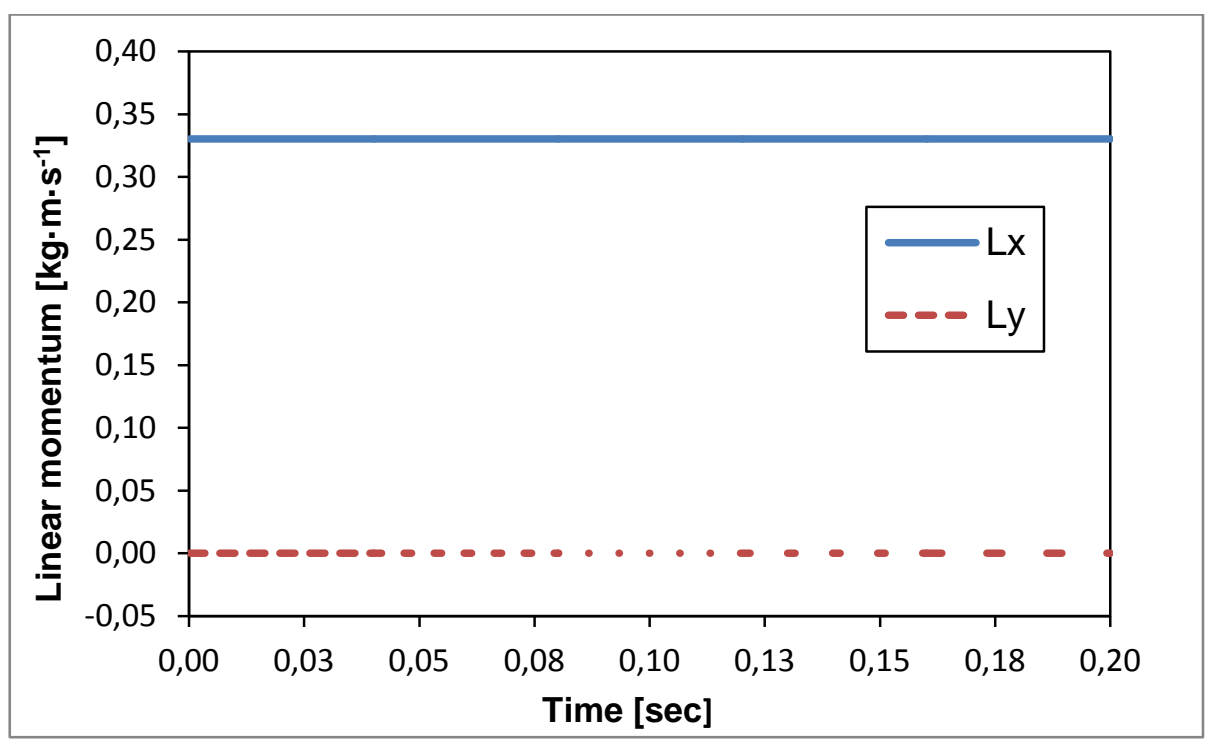

Figure 9. Impact of two aligned elastic hollow cylinders sliding on a frictionless rigid surface. Linear momentum evolution

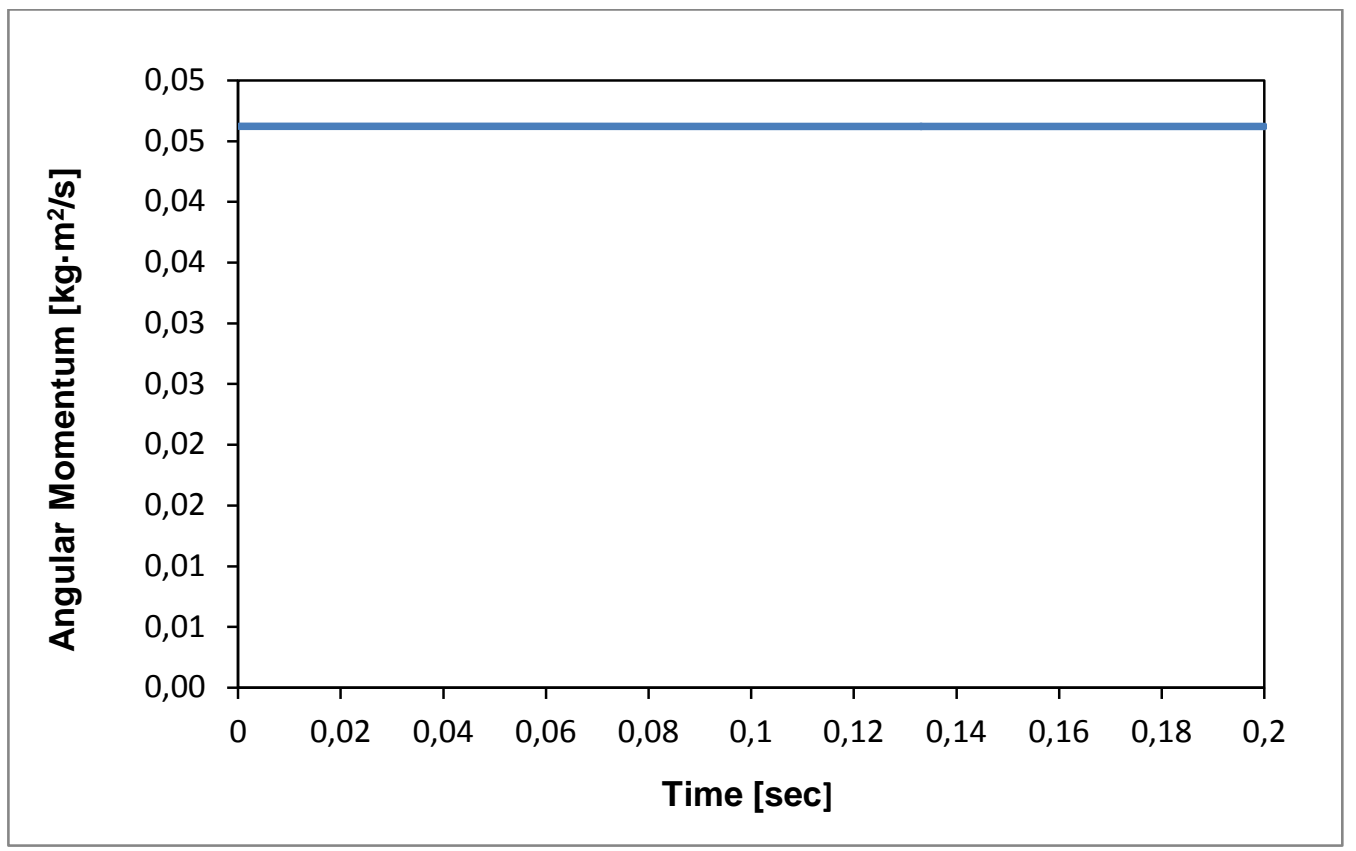

Figure 10. Impact of two aligned elastic hollow cylinders sliding on a frictionless rigid surface. Angular momentum evolution 


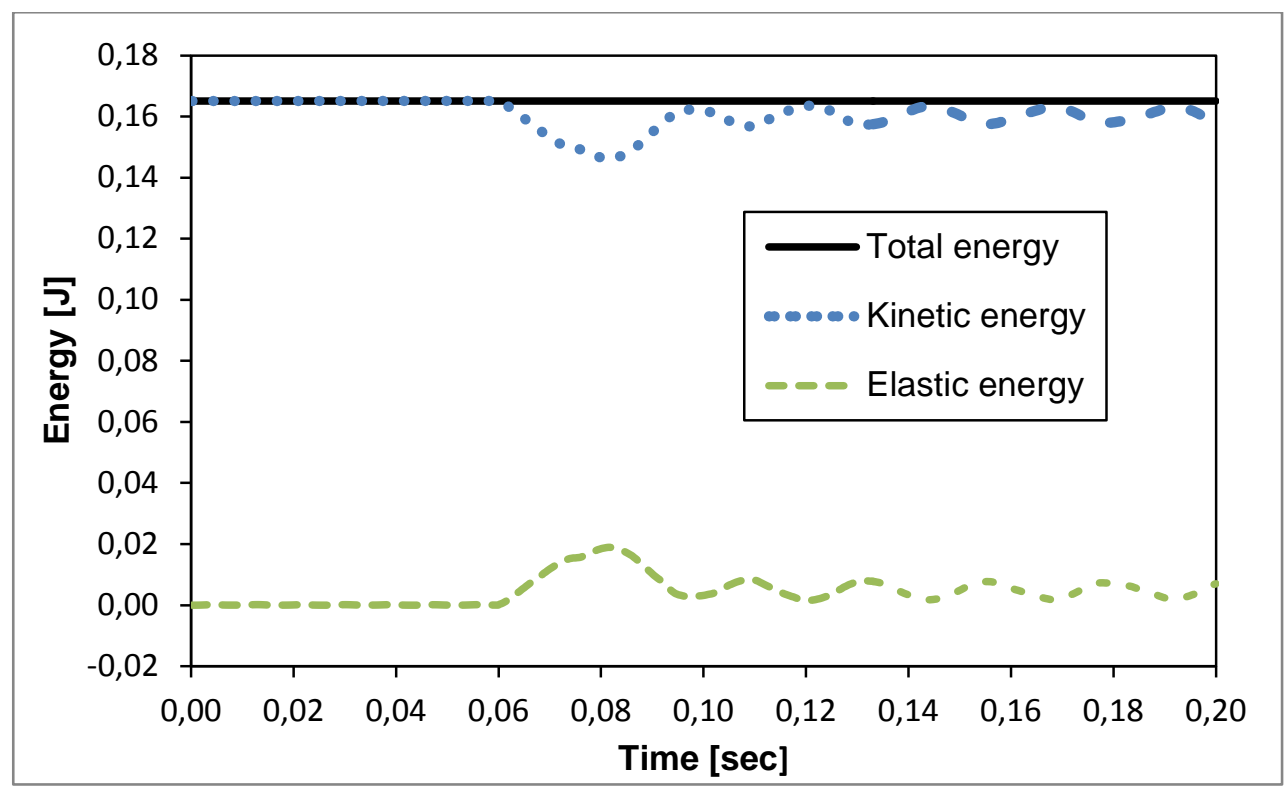

Figure 11. Impact of two aligned elastic hollow cylinders sliding on a frictionless rigid surface. Evolution of the different energies.

\subsection{Elastic ball bouncing on a rigid rough surface}

This example deals with the numerical simulation of an elastic ball bouncing on a rigid surface. The ball is a spherical crown with internal radius of $0.20 \mathrm{~m}$ and external radius of $0.40 \mathrm{~m}$. An elastic Saint-Venant material model with Young's elastic modulus of 4.0E+06 Pa and Poisson's coefficient of 0.45 is used. The mass density of the ball is $1000 \mathrm{Kg} / \mathrm{m}^{3}$. Only the self-weight of the ball is considered as loading conditions. The center of the ball is initially located at $1 \mathrm{~m}$ of the rigid surface. An initial velocity of $2.0 \mathrm{~m} / \mathrm{sec}$ in the y-direction is considered. Full stick contact conditions are considered.

The ball has been discretized using standard Galerkin P1 linear displacement tetrahedral finite elements. The finite element mesh used for the discretization of the ball consists of 8299 elements and 1571 nodes. The rigid surface has been discretized using 4 shell elements. All the degrees of freedom of the shell elements have been prescribed to zero. Time increments of 0.001 secs have been used. Figure 12 shows the geometry and finite element meshes used in the numerical simulation.

Figure 13 shows the deformed configurations of the ball at different time steps. It can be observed that the ball impact the rigid surface at the time steps $0.42 \mathrm{sec}, 1.25 \mathrm{sec}$ and $2.01 \mathrm{sec}$. Due to the full stick contact condition, a rotation of the ball takes place when it impacts on the rigid surface. The angular momentum of the ball is increasing each time it contacts the rigid surface. It can be also observed that the translation velocity of the ball is decreasing in order to keep constant the kinetic energy.

Figure 14 shows the time evolution of the total, kinetic, elastic and potential energies. Despite the fact that a full stick contact condition has been considered, the total energy is conserved. This is due to the fact that the work done by the frictional tractions is zero, because 
the frictional slip is zero due to the full stick contact condition. This results in a perfect rotation of the ball on the rigid surface, without any slip motion.

It can be also observed that different effects take place every time the ball comes into contact with the rigid surface. The kinetic energy is increasing due to the elastic vibration of the ball generated by the impact. Similarly, the potential energy is decreasing because the ball is falling due to the increase of the elastic energy. It can be also observed a slight decrease of the maximum kinetic energy, being also compatible with the increase of the elastic energy.
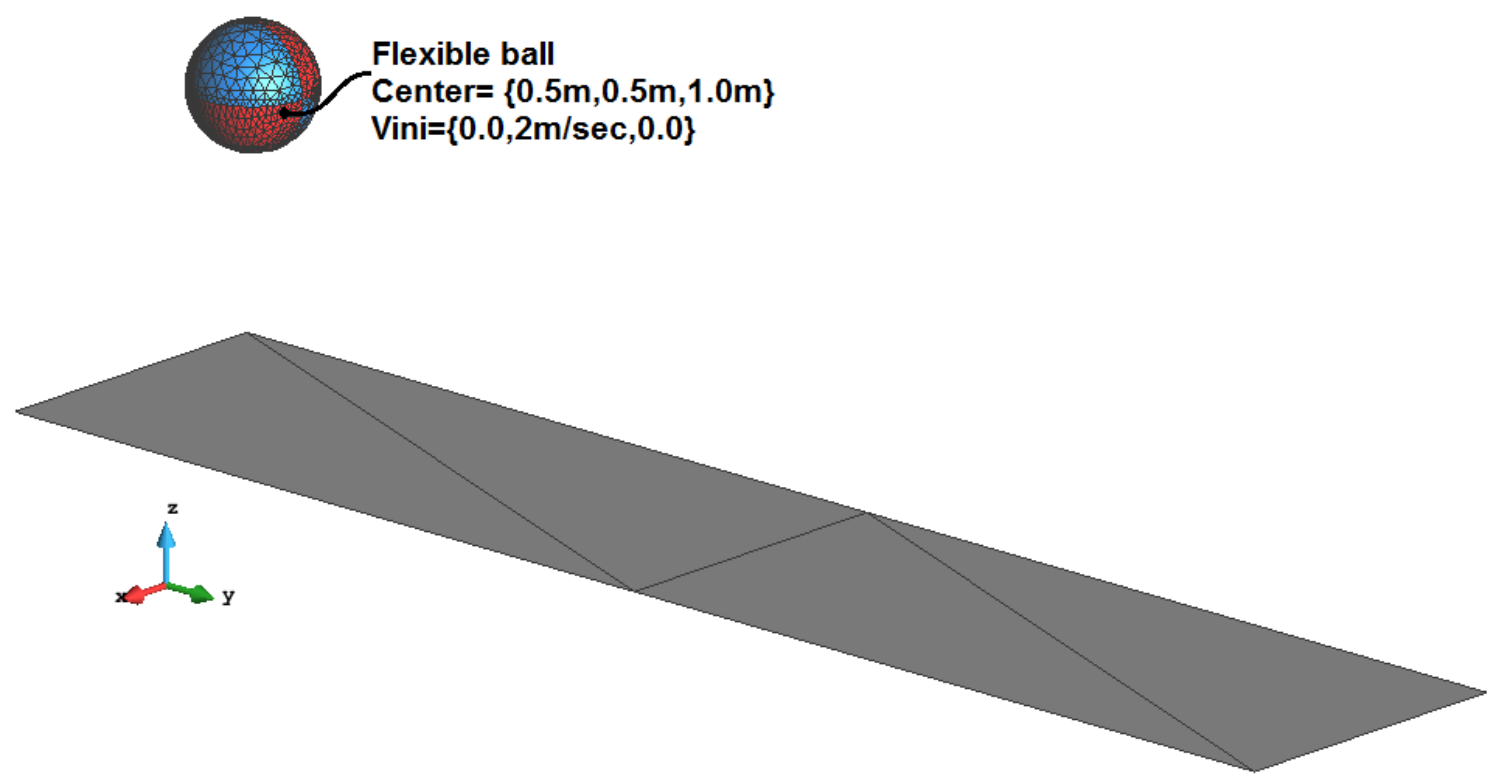

Figure 12. Elastic ball bouncing on a rigid surface. Geometry and finite element meshes. 


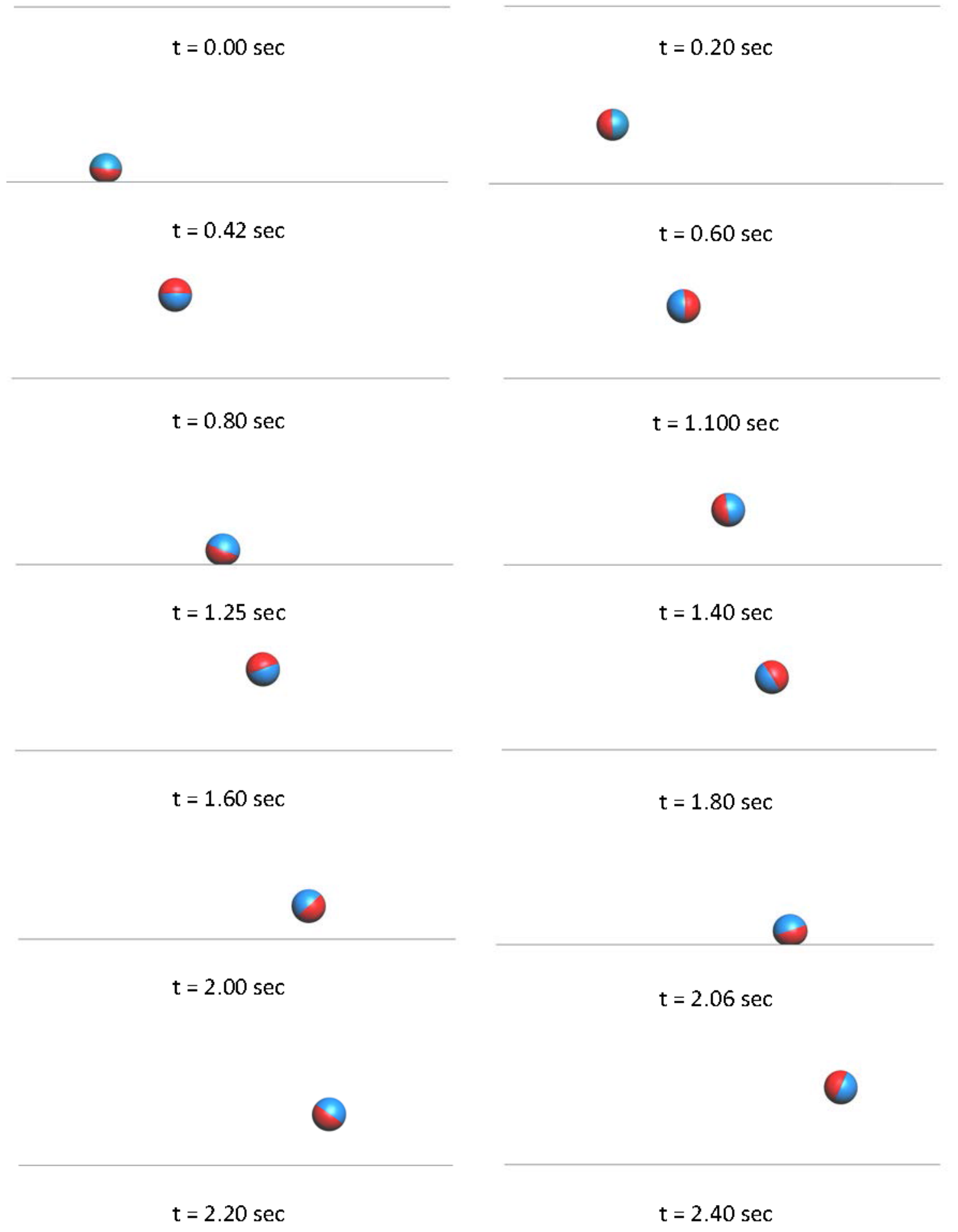

Figure 13. Elastic ball bouncing on a rigid surface. Deformation configuration at different time steps. 


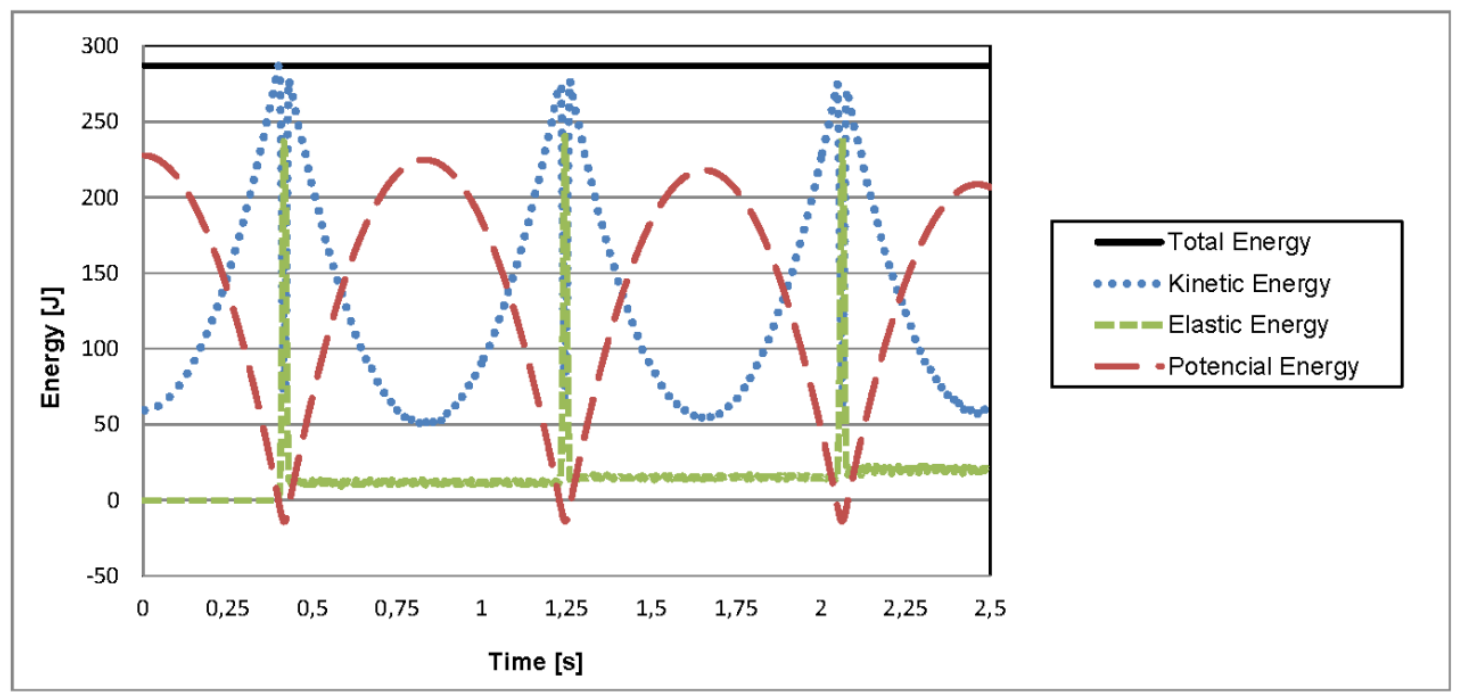

Figure 14. Elastic ball bouncing on a rigid surface. Evolution of the different energies.

\section{Conclusions}

The focus of this work has been placed on energy-momentum conserving schemes formulated within the framework of the direct elimination method. Energy-momentum conservation properties have been addressed for two particular cases of interest in dynamic contact problems using the DEA: full stick friction and slip frictionless. For the full stick frictional case the main goal was to show that the resulting algorithm was inheriting the energy conservation property shown at the continuum level. For the slip frictionless case the goal was to show that the resulting algorithm was inheriting both the energy and momentum conservation properties shown at the continuum level. Note that, as those conservation properties do not hold under slip frictional conditions, this case has not been considered and its formulation within the framework of the DEA is out of the scope of interest of this work.

Constraints arising in full stick and frictionless contact problems have been imposed in a strong fashion by a direct elimination of the involved degrees of freedom of the resulting system of equations. A more convenient and natural formulation of the direct elimination method for the slip frictionless case has been derived. This formulation has been given in terms of the contravariant components of the iso-parametric coordinates of the incremental slip of the closest-point-projection on the covariant basis vectors evaluated at the mid-point configuration.

An assessment of the performance of the resulting algorithms has been shown in a number of selected dynamic contact numerical examples, under full stick friction and slip frictionless conditions. The first two examples deal with the numerical simulation of the dynamic impact of three aligned quasi-rigid cylinders sliding on a frictionless rigid surface, and the numerical simulation of the dynamic impact of two elastic hollow cylinders sliding on a frictionless rigid surface. Here the goal was to show the conservation of the key discrete properties (total linear momentum, total angular momentum, and total energy) exhibited by the time stepping contact algorithm proposed. It has been shown that conservation of those key discrete properties exhibited at the continuum level, are also preserved by the time stepping algorithm. The last example deals with the numerical simulation of an elastic ball bouncing on a 
rough rigid surface. Full stick friction conditions were considered. Here the goal was to show that the proposed contact formulation exhibits the conservation of the discrete total energy. It has been shown that discrete total energy is fully conserved by the time stepping algorithm.

\section{Acknowledgements}

Authors would like to acknowledge COMPASS for providing the code RamSeries where the contact algorithms shown in this work have been implemented. Partial financial support by the Ministerio de Economía y Competitividad, through the project RTC-2016-4826-5 "Desarrollo de nuevos métodos y herramientas para la optimización del proceso de fabricación de envases de vidrio (MOVASE)" is also acknowledged.

\section{Appendix 1}

This Appendix contents the computation of the variations of the covariant tangent vectors $\tau_{\xi, n+\alpha}$ and $\tau_{\eta, n+\alpha}$ at the configuration $n+\alpha$ of the closest-point-projection on the master surface.

Covariant tangent vectors $\tau_{\xi, n+\alpha}$ and $\tau_{\eta, n+\alpha}$ to the isoparametric coordinates at the closest-point-projection at the configuration $n+\alpha$ are defined as,

$$
\boldsymbol{\tau}_{\xi, n+\alpha}:=\mathbf{N}_{\xi, n+\alpha} \mathbf{x}_{m, n+\alpha}, \quad \boldsymbol{\tau}_{\eta, n+\alpha}:=\mathbf{N}_{\eta, n+\alpha} \mathbf{x}_{m, n+\alpha}
$$

where the $n_{\mathrm{dim}} \times n_{\mathrm{dim}} n_{\text {mnod }}$ matrices $\mathbf{N}_{\xi, n+\alpha}, \mathbf{N}_{\eta, n+\alpha}$ are given by,

$$
\begin{aligned}
& \mathbf{N}_{\xi, n+\alpha}:=\left[\mathbf{N}_{\xi_{1}, n+\alpha}, \ldots, \mathbf{N}_{\xi_{\text {mnod }}, n+\alpha}\right], \quad \mathbf{N}_{\xi_{A}, n+\alpha}:=\left.\frac{\partial N_{A}(\boldsymbol{\xi})}{\partial \xi}\right|_{\bar{\xi}_{n+\alpha}} \mathbf{1} \\
& \mathbf{N}_{\eta, n+\alpha}:=\left[\mathbf{N}_{\eta_{1}, n+\alpha}, \ldots, \mathbf{N}_{\eta_{\text {mnod }}, n+\alpha}\right], \quad \mathbf{N}_{\eta_{A}, n+\alpha}:=\left.\frac{\partial N_{A}(\boldsymbol{\xi})}{\partial \eta}\right|_{\bar{\xi}_{n+\alpha}} \mathbf{1}
\end{aligned}
$$

The variation of the covariant tangent vectors $\tau_{\xi, n+\alpha}$ and $\tau_{\eta, n+\alpha}$ given by (87) takes the form,

$$
\begin{aligned}
\Delta \tau_{\xi, n+\alpha} & =\mathbf{N}_{\xi, n+\alpha} \Delta \mathbf{u}_{m, n+\alpha}+\Delta \mathbf{N}_{\xi, n+\alpha} \mathbf{x}_{m, n+\alpha} \\
\Delta \tau_{\eta, n+\alpha} & =\mathbf{N}_{\eta, n+\alpha} \Delta \mathbf{u}_{m, n+\alpha}+\Delta \mathbf{N}_{\eta, n+\alpha} \mathbf{x}_{m, n+\alpha}
\end{aligned}
$$

where the variations $\Delta \mathbf{N}_{\xi, n+\alpha}, \Delta \mathbf{N}_{\eta, n+\alpha}$ are given by,

$$
\begin{aligned}
\Delta \mathbf{N}_{\xi, n+\alpha} & =\mathbf{N}_{\xi \xi, n+\alpha} \Delta \bar{\xi}_{n+\alpha}+\mathbf{N}_{\xi \eta, n+\alpha} \Delta \bar{\eta}_{n+\alpha} \\
\Delta \mathbf{N}_{\eta, n+\alpha} & =\mathbf{N}_{\eta \xi, n+\alpha} \Delta \bar{\xi}_{n+\alpha}+\mathbf{N}_{\eta \eta, n+\alpha} \Delta \bar{\eta}_{n+\alpha}
\end{aligned}
$$

where the $n_{\text {dim }} \times n_{\text {dim }} n_{\text {mnod }}$ matrix operators $\mathbf{N}_{\xi \xi, n+\alpha}, \mathbf{N}_{\xi \eta, n+\alpha}=\mathbf{N}_{\eta \xi, n+\alpha}, \mathbf{N}_{\eta \eta, n+\alpha}$ are defined as, 


$$
\begin{aligned}
& \mathbf{N}_{\xi \xi, n+\alpha}:=\left[\mathbf{N}_{\xi \xi_{1}, n+\alpha}, \ldots, \mathbf{N}_{\xi \xi_{\text {mnod }}, n+\alpha}\right], \quad \mathbf{N}_{\xi \xi_{A}, n+\alpha}:=\left.\frac{\partial^{2} N_{A}(\boldsymbol{\xi})}{\partial \xi \partial \xi}\right|_{\bar{\xi}_{n+\alpha}} \mathbf{1} \\
& \mathbf{N}_{\xi \eta, n+\alpha}:=\left[\mathbf{N}_{\xi \eta_{1}, n+\alpha}, \ldots, \mathbf{N}_{\xi \eta_{\text {mnod }}, n+\alpha}\right], \quad \mathbf{N}_{\xi \eta_{A}, n+\alpha}:=\left.\frac{\partial^{2} N_{A}(\boldsymbol{\xi})}{\partial \xi \partial \eta}\right|_{\bar{\xi}_{n+\alpha}} \mathbf{1} \\
& \mathbf{N}_{\eta \eta, n+\alpha}:=\left[\mathbf{N}_{\eta \eta_{1}, n+\alpha}, \ldots, \mathbf{N}_{\eta \eta_{\text {mnod }}, n+\alpha}\right], \quad \mathbf{N}_{\eta \eta_{A}, n+\alpha}:=\left.\frac{\partial^{2} N_{A}(\boldsymbol{\xi})}{\partial \eta \partial \eta}\right|_{\overline{\boldsymbol{\xi}}_{n+\alpha}} \mathbf{1}
\end{aligned}
$$

Substituting (90) into (89), the variation of the covariant tangent vectors $\tau_{\xi, n+\alpha}$ and $\tau_{\eta, n+\alpha}$ given by (89) takes the form,

$$
\begin{aligned}
& \Delta \boldsymbol{\tau}_{\xi, n+\alpha}=\mathbf{N}_{\xi, n+\alpha} \Delta \mathbf{u}_{m, n+\alpha}+\mathbf{N}_{\xi \xi, n+\alpha} \mathbf{x}_{m, n+\alpha} \Delta \bar{\xi}_{n+\alpha}+\mathbf{N}_{\xi \eta, n+\alpha} \mathbf{x}_{m, n+\alpha} \Delta \bar{\eta}_{n+\alpha} \\
& \Delta \tau_{\eta, n+\alpha}=\mathbf{N}_{\eta, n+\alpha} \Delta \mathbf{u}_{m, n+\alpha}+\mathbf{N}_{\eta \xi, n+\alpha} \mathbf{x}_{m, n+\alpha} \Delta \bar{\xi}_{n+\alpha}+\mathbf{N}_{\eta \eta, n+\alpha} \mathbf{x}_{m, n+\alpha} \Delta \bar{\eta}_{n+\alpha}
\end{aligned}
$$

and taking into account that $\Delta \mathbf{u}_{m, n+\alpha}=\alpha \Delta \mathbf{u}_{m, n+1}, \Delta \bar{\xi}_{n+\alpha}=\alpha \Delta \bar{\xi}_{n+1}$ and $\Delta \bar{\eta}_{n+\alpha}=\alpha \Delta \bar{\eta}_{n+1}$ the variation of the covariant tangent vectors $\tau_{\xi, n+\alpha}$ and $\tau_{\eta, n+\alpha}$ given by (89) takes the form,

$$
\begin{aligned}
& \Delta \boldsymbol{\tau}_{\xi, n+\alpha}=\alpha\left(\mathbf{N}_{\xi, n+\alpha} \Delta \mathbf{u}_{m, n+1}+\mathbf{N}_{\xi \xi, n+\alpha} \mathbf{x}_{m, n+1} \Delta \bar{\xi}_{n+1}+\mathbf{N}_{\xi \eta, n+\alpha} \mathbf{x}_{m, n+\alpha} \Delta \bar{\eta}_{n+1}\right) \\
& \Delta \tau_{\eta, n+\alpha}=\alpha\left(\mathbf{N}_{\eta, n+\alpha} \Delta \mathbf{u}_{m, n+1}+\mathbf{N}_{\eta \xi, n+\alpha} \mathbf{x}_{m, n+\alpha} \Delta \bar{\xi}_{n+1}+\mathbf{N}_{\eta \eta, n+\alpha} \mathbf{x}_{m, n+\alpha} \Delta \bar{\eta}_{n+1}\right)
\end{aligned}
$$

Note that for linear elements, the matrix operators given by (91) are zero, and thus the variations of the matrices given by (91) is zero, yielding the following simplified expressions for the variations of the covariant tangent vectors $\tau_{\xi, n+\alpha}$ and $\tau_{\eta, n+\alpha}$,

$$
\begin{aligned}
& \Delta \tau_{\xi, n+\alpha}=\mathbf{N}_{\xi, n+\alpha} \Delta \mathbf{u}_{m, n+\alpha}=\alpha \mathbf{N}_{\xi, n+\alpha} \Delta \mathbf{u}_{m, n+1} \\
& \Delta \tau_{\eta, n+\alpha}=\mathbf{N}_{\eta, n+\alpha} \Delta \mathbf{u}_{m, n+\alpha}=\alpha \mathbf{N}_{\eta, n+\alpha} \Delta \mathbf{u}_{m, n+1}
\end{aligned}
$$

\section{References}

1. Agelet de Saracibar C. A new frictional time integration algorithm for large slip multi-body frictional contact problems, Computer Methods in Applied Mechanics and Engineering 1997; 142: 303-334.

2. Agelet de Saracibar C. Numerical analysis of coupled thermomechanical contact problems. Computational model and applications, Archives of Computational Methods in Mechanics 1998; 5: 243-301. doi: 10.1007/BF02897875.

3. Armero F, Petocz E. Formulation and analysis of conserving algorithms for frictionless dynamic contact/impact problems, Computer Methods in Applied Mechanics and Engineering 1998; 158: 269-300.

4. Armero F, Petocz E. A new dissipative time-stepping algorithm for frictional contact problems: Formulation and analysis, Computer Methods in Applied Mechanics and Engineering 1999; 179: 151-178. 
5. Armero F, Romero I. On the formulation of high-frequency dissipative time-stepping algorithms for nonlinear dynamics. Part I: low-order methods for two model problems and nonlinear elastodynamics, Computer Methods in Applied Mechanics and Engineering 2001; 190: $2603-2649$.

6. Armero F. Energy-Momentum Algorithms for the Nonlinear Dynamics of Elastoplastic Solids, IUTAM Symposium on Theoretical, Computational and Modelling Aspects of Inelastic Media, IUTAM Book Series, Volume 11, 2008, pp. 251-262.

7. Betsch P, Hesch C. Energy-momentum conserving schemes for frictionless dynamic contact problems. Part I: NTS method. In: Wriggers P, Nackenhorst U. (eds.) IUTAM symposium on computational methods in contact mechanics, vol 3 of IUTAM Bookseries, Springer, Berlin, 2007, pp. 77-96

8. Brunssen S, Hartmann S, Ramm E, Wohlmuth B. Unilateral non-linear dynamic contact of thin-walled structures using a primal-dual active set strategy, International Journal for Numerical Methods Engineering 2007; 70: 883-912.

9. Di Capua D, Agelet de Saracibar C. A direct elimination algorithm for quasi-static and dynamic contact problems, Finite Elements in Analysis and Design 2015; 93: 107-125.

10. Duvaut G, Lions JL. Les Inequations en Mecanique et en Physique, Dunod, Paris, 1972.

11. El-Abbasi N, Bathe KJ. Stability and patch test performace of contact discretizations and a new solution algorithm, International Journal for Numerical Methods in Engineering 2001; 79:1473-1486.

12. Fischer KA, Wriggers P. Frictionless $2 d$ contact formulations for finite deformations based on the mortar method. Computational Mechanics 2005, 36:226-244

13. Flemish B, Puso MA, Wohlmuth BI. A new dual mortar method for curved interfaces: 2delasticity. International Journal for Numerical Methods in Engineering 2005, 63:813-832.

14. Gonzalez O. Exact energy-momentum conserving algorithms for general models in nonlinear elasticity, Computer Methods in Applied Mechanics and Engineering 2000; 190: 1763-1783.

15. Haikal G, Hjelmstad KD. A finite element formulation of non-smooth contact based on oriented volumes for quadrilateral and hexahedral elements. Computer Methods in Applied Mechanics and Engineering 2007, 196:4690-4711

16. Hauret P, LeTallec P. Energy-controlling time integration methods for nonlinear elastodynamics and low-velocity impact. Computer Methods in Applied Mechanics and Engineering 2006; 195:4890-4916.

17. Hesch C, Betsch P. Transient three-dimensional contact problems: mortar method. Mixed methods and conserving integration. Computational Mechanics 2011; 48(4):461-475

18. Hesch C, Betsch P. Transient 3d contact problems-NTS method: mixed methods and conserving integration. Computational Mechanics 2011; 48(4):437-449

19. Hesch C, Betsch P. Transient 3d domain decomposition problems: frame-indifferent mortar constraints and conserving integration. International Journal for Numerical Methods in Engineering 2010; 82:329-358.

20. Hesch C, Betsch P. A mortar method for energy-momentum conserving schemes in frictionless dynamic contact problems. International Journal for Numerical Methods in Engineering 2009; 77:1468-1500.

21. Hüeber S, Wohlmuth B. A primal-dual active set strategy for non-linear multibody contact problems, Computer Methods in Applied Mechanics and Engineering 2005; 194: 31473155.

22. Kikuchi N, Oden JT. Contact Problems in Elasticity: A Study of Variational Inequalities and Finite Element Methods, SIAM, Philadelphia, 1988. 
23. Laursen TA. Formulation and Treatment of Frictional Contact Problems Using Finite Elements, Ph.D. Dissertation, Department of Mechanical Engineering, Stanford University, 1992.

24. Laursen TA, Chawla V. Design of energy conserving algorithms for frictionless dynamic contact problems, International Journal for Numerical Methods in Engineering 1997; 40: 863-886.

25. Laursen TA, Love GR. Improved implicit integrators for transient impact problems geometric admissibility within the conserving framework. International Journal for Numerical Methods in Engineering 2002, 53:245-274

26. Laursen TA. Computational Contact and Impact Mechanics, Springer, 2002

27. McDevitt TW, Laursen TA. A mortar-finite element formulation for frictional contact problems. International Journal for Numerical Methods in Engineering 2000; 48: 15251547.

28. Papadopoulos P, Taylor RL. A mixed formulation for the finite element solution of contact problems, UCB/SEMM Report 90/18, University of California, Berkeley, 1990.

29. Popp A, Gitterle M, Gee MW, Wall WA. A dual mortar approach for 3D finite deformation contact with consistent linearization. International Journal for Numerical Methods in Engineering 2010, 83:1428-1465.

30. Puso MA, Laursen TA. A mortar segment-to-segment contact method for large deformation solid mechanics, Computer Methods in Applied Mechanics and Engineering 2004; 193 : 601-629.

31. Puso MA, Laursen TA. A mortar segment-to-segment frictional contact method for large deformations, Computer Methods in Applied Mechanics and Engineering 2004; 193: 48914913.

32. Puso MA, Laursen TA, Solberg JM (2008) A segment-to-segment mortar contact method for quadratic elements and large deformations. Computer Methods in Applied Mechanics and Engineering 2008; 197: 555-566.

33. RamSeries code, http://www.compassis.com/compass/en/Productos/RamSeries.

34. Simo JC, Tarnow N. The discrete energy-momentum method. Conserving algorithm for non-linear elastodynamics, ZAMP 1992; 43: 757-793.

35. Simo JC, Tarnow N, Wong KK. Exact energy-momentum conserving algorithms and symplectic schemes for nonlinear dynamics, Computer Methods in Applied Mechanics and Engineering 1992; 1: 63-116.

36. Simo JC, Tarnow N. A new energy and momentum conserving algorithm for the non-linear dynamics of shells, International Journal for Numerical methods in Engineering 1994; 37: 2525-2550.

37. Simo JC, Tarnow N, Doblaré M. Non-linear dynamics of three-dimensional rods: exact energy and momentum conserving algorithms, International Journal for Numerical Methods in Engineering 1995; 38: 1431-1474.

38. Truesdell CA, Noll WM. The Nonlinear Field Theories of Mechanics, Handbuch der Physik, vol. III/3, Springer, Berlin, 1965.

39. Wohlmuth B. A mortar finite element method using dual spaces for the Lagrange multiplier, SIAM Journal Numerical Analysis 2000; 38: 989-1012

40. Wriggers P. Computational Contact Mechanics, Springer, 2006.

41. Yang B. Mortar Finite Element Methods for Large Deformation Contact Mechanics, Ph.D. Dissertation, Duke University, 2006. 